TITLE:

\title{
Visuomotor signals for reaching movements in the rostro-dorsal sector of the monkey thalamic reticular nucleus
}

\section{AUTHOR(S):}

Saga, Yosuke; Nakayama, Yoshihisa; Inoue, Ken-ichi; Yamagata, Tomoko; Hashimoto, Masashi; Tremblay, Léon; Takada, Masahiko; Hoshi, Eiji

\section{CITATION:}

Saga, Yosuke ...[et al]. Visuomotor signals for reaching movements in the rostro-dorsal sector of the monkey thalamic reticular nucleus. European Journal of Neuroscience 2017, 45(9): 1186-1199

\section{ISSUE DATE:}

2017-05

URL:

http://hdl.handle.net/2433/224995

\section{RIGHT:}

This is the peer reviewed version of the following article: [Saga, Y., Nakayama, Y., Inoue, K.-i., Yamagata, T., Hashimoto, M., Tremblay, L., Takada, M., Hoshi, E. (2017), Visuomotor signals for reaching movements in the rostro-dorsal sector of the monkey thalamic reticular nucleus. European Journal of Neuroscience, 45: 1186-1199], which has been published in final form at https://doi.org/10.1111/ejn.13421. This article may be used for non-commercial purposes in accordance with Wiley Terms and Conditions for Self-Archiving: The full-text file will be made open to the public on 26 APR 2018 in accordance with publisher's 'Terms and Conditions for Self-Archiving'.; この論文は出版社版でありません。引用の際に は出版社版をご確認ご利用ください。; This is not the published version. Please cite only the published version. 


\title{
Visuomotor signals for reaching movements in the rostro-dorsal sector of the monkey thalamic reticular nucleus
}

\author{
Yosuke Saga, ${ }^{1,2,3}$ Yoshihisa Nakayama, ${ }^{1,3}$ Ken-ichi Inoue, ${ }^{4}$ Tomoko Yamagata, ${ }^{1,3}$ Masashi Hashimoto, ${ }^{1,3}$ \\ Léon Tremblay, ${ }^{2}$ Masahiko Takada ${ }^{4,5}$ and Eiji Hoshi, ${ }^{1,3,5}$ \\ ${ }^{1}$ Frontal Lobe Function Project, Tokyo Metropolitan Institute of Medical Science, Tokyo, Japan \\ ${ }^{2}$ Centre de Neuroscience Cognitive, UMR-5229 CNRS, 67 Boulevard Pinel, 69675 Bron Cedex, France \\ ${ }^{3}$ Tamagawa University Brain Science Institute, Tokyo, Japan \\ ${ }^{4}$ Systems Neuroscience Section, Primate Research Institute, Kyoto University, Inuyama, Aichi, Japan \\ ${ }^{5}$ AMED-CREST, Japan Agency for Medical Research and Development, Tokyo, Japan
}

Keywords: action, nonhuman primates, premotor cortex, reaching movement, visuomotor control

Edited by Helen Barbas

Received 11 July 2016, revised 28 September 2016, accepted 29 September 2016

\begin{abstract}
The thalamic reticular nucleus (TRN) collects inputs from the cerebral cortex and thalamus and, in turn, sends inhibitory outputs to the thalamic relay nuclei. This unique connectivity suggests that the TRN plays a pivotal role in regulating information flow through the thalamus. Here, we analyzed the roles of TRN neurons in visually guided reaching movements. We first used retrograde transneuronal labeling with rabies virus, and showed that the rostro-dorsal sector of the TRN (TRNrd) projected disynaptically to the ventral premotor cortex (PMv). In other experiments, we recorded neurons from the TRNrd or PMv while monkeys performed a visuomotor task. We found that neurons in the TRNrd and PMv showed visual-, set-, and movement-related activity modulation. These results indicate that the TRNrd, as well as the PMv, is involved in the reception of visual signals and in the preparation and execution of reaching movements. The fraction of neurons that were non-selective for the location of visual signals or the direction of reaching movements was greater in the TRNrd than in the PMv. Furthermore, the fraction of neurons whose activity increased from the baseline was greater in the TRNrd than in the PMv. The timing of activity modulation of visualrelated and movement-related neurons was similar in TRNrd and PMv neurons. Overall, our data suggest that TRNrd neurons provide motor thalamic nuclei with inhibitory inputs that are predominantly devoid of spatial selectivity, and that these signals modulate how these nuclei engage in both sensory processing and motor output during visually guided reaching behavior.
\end{abstract}

\section{Introduction}

The motor areas in the cerebral cortex have reciprocal connections with the motor thalamus. Situated around the thalamic relay nuclei, including the motor thalamus, is the thalamic reticular nucleus (TRN), a thin, sheet-like cluster of GABAergic neurons that circumscribes thalamic nuclei laterally (Houser et al., 1980; Guillery \& Sherman, 2002; Jones, 2007). The TRN receives collateral inputs from the thalamic neurons projecting to the cerebral cortex and cortical neurons projecting to the thalamus (Chow, 1952; Schell \& Strick, 1984; Cicirata et al., 1990; Gonzalo-Ruiz \& Lieberman, 1995a,b; Pinault \& Deschenes, 1998; Rouiller et al., 1998; Crabtree \& Isaac, 2002). Additionally, the TRN receives widespread inputs from the basal ganglia and cholinergic and noradrenergic nuclei (Carman et al., 1964; Jones, 1975; Morrison \& Foote, 1986; Pare et al., 1990; Hazrati \& Parent, 1991; McCormick, 1992; Gandia et al., 1993; Asanuma, 1994;

Correspondence: Dr Y. Saga, ${ }^{2}$ Centre de Neuroscience Cognitive, as above. E-mail: sagalesvins@gmail.com

Steriade, 2005). In turn, TRN neurons send GABAergic, inhibitory outputs to the thalamic relay nuclei. This anatomical organization suggests that the TRN plays an important role in motor control.

The functional role(s) of the TRN in movement remain(s) unknown because TRN neurons have rarely been studied in terms of motor control. In cats, TRN neurons in the somatosensory sector are active during walking, and the activation phase is dependent on the receptive fields (RFs) (Marlinski et al., 2012; Marlinski \& Beloozerova, 2014). In monkeys, neurons in the dorsal portion of the TRN respond to somatosensory stimulation (Pollin \& Rokyta, 1982). However, neuronal activity has not fully been examined in task-performing monkeys, except in a series of studies showing that neuronal activity in the visual sector of the TRN is modulated dynamically by visual attention (McAlonan et al., 2006, 2008).

To investigate the contribution of the TRN to motor control, we sought to characterize TRN neurons in monkeys while they were performing a visually guided reaching movements. We integrated neuroanatomical and electrophysiological approaches. In the 
neuroanatomical approach, we used retrograde transneuronal labeling with rabies virus to identify a TRN sector containing neurons that projected across synapses to the ventral premotor cortex (PMv) (Kelly \& Strick, 2000; Saga et al., 2011; Takahara et al., 2012). The PMv has been shown to play a central role in visually guided reaching movement (Kurata \& Hoffman, 1994; Kurata \& Hoshi, 2002; Hoshi \& Tanji, 2006; Yamagata et al., 2009). We found that the rostro-dorsal sector of the TRN (TRNrd) contained neurons disynaptically projecting to PMv. In the physiological approach, we analyzed neurons in this portion of the TRN in monkeys while they performed a visually guided target-reaching task. Furthermore, we compared the response properties of TRNrd neurons with those of PMv neurons in other monkeys performing the reaching task. Our results indicate that TRN neurons exhibit a range of responses, such as visual-, set-, and movement-related activity. This suggests that the TRN sends inhibitory signals to the thalamic relay nuclei to modulate activities at multiple time points in the generation of reaching movements.

\section{Materials and methods}

\section{Animals and experimental conditions}

Six monkeys (Macaca fuscata; five males, $5.0-8.5 \mathrm{~kg}$; one female, $5.0 \mathrm{~kg}$ ) were used in this study. Four monkeys (Monkeys 1-4) were used for TRN neuron recordings (Monkeys 1 and 2; Saga et al., 2013) and PMv neuron recordings (Monkeys 3 and 4; Yamagata et al., 2009). The other two monkeys (Monkeys 5 and 6) were used for investigating anatomical connectivity of TRN with PMv. Our prior report included results obtained with Monkeys 5 and 6, which showed the origins of multisynaptic projections from the basal ganglia to the PMv (Ishida et al., 2016).

All experiments were conducted in accordance with the National Institutes of Health guidelines and the guidelines of the Tokyo Metropolitan Institute of Medical Science, Tamagawa University, and the Primate Research Institute at Kyoto University. All animal care and experimental procedures were approved by the Animal Care and Use Committee of Tokyo Metropolitan Institute of Medical Science, Tamagawa University, and the Primate Research Institute at Kyoto University.

\section{Electrophysiological study}

During the experimental sessions for neuronal recording from taskperforming monkeys (Monkeys 1-4), each monkey sat in a primate chair with its head and left arm restrained. A button was installed at waist level in front of the chair, where the monkey could readily press and release it with the right hand. A 19-inch color video monitor equipped with a touch-sensitive screen was placed in front of the monkey (30 $\mathrm{cm}$ from the eyes). Eye movement was monitored at $120 \mathrm{~Hz}$ using an infrared eye-tracking system (resolution, $0.25^{\circ}$ visual angle; RHS-M; Applied Science Laboratories, Spokane, USA). The TEMPONET system (Reflective Computing) was used to control the behavioral task, to regulate the opening and closing of a solenoid valve serially installed in the reward delivery system, and to collect data, at $1000 \mathrm{~Hz}$. A liquid reward (apple juice) was delivered via a stainless-steel tube placed in front of the monkey's mouth.

\section{Surgery and electrophysiological recordings}

Aseptic surgery was performed under pentobarbital sodium anesthesia $(20-25 \mathrm{mg} / \mathrm{kg}$, intravenously) following the induction of anesthesia using ketamine hydrochloride $(10 \mathrm{mg} / \mathrm{kg}$, intramuscularly) and atropine sulfate. Antibiotics and analgesics were used to prevent postsurgical infection and pain respectively. Polycarbonate and titanium screws were implanted in the skull, and two plastic pipes were firmly attached using acrylic resin. Part of the skull over the left frontal lobe was removed, and a recording chamber was implanted to permit access to the TRN, thalamic relay nuclei, and the globus pallidus in two monkeys (Monkeys 1 and 2), and the $\mathrm{PMv}$, dorsal premotor cortex (PMd), and primary motor cortex (M1) in two other monkeys (Monkeys 3 and 4).

Neuronal activity was recorded using a glass-insulated tungsten microelectrode or glass-insulated Elgiloy-alloy mictoelectrodes (0.5$2.0 \mathrm{M} \Omega$ at $1 \mathrm{kHz}$ ) inserted into the brain through a 23-gauge guide tube that penetrated the dura mater. A hydraulic microdrive (MO972 or MO-81-S, Narishige, Tokyo Japan) was used to move the electrode using a micrometer step. Single-unit potentials were amplified using a multichannel processor and sorted using a multi-spike detector (MCP and ASD, Alpha Omega Engineering, Nazareth, Israel). Recording sites were verified by examining magnetic resonance images (1.5 T Sonata; Siemens, Erlangen, Germany).

To identify the PMv, we checked the neuronal responses to somatosensory and visual stimuli, and applied intracortical microstimulation (ICMS) through the tip of an inserted electrode (11-44 pulses of $200 \mu$ s width at $333 \mathrm{~Hz}$; current, $<50 \mu \mathrm{A}$; see detail in Yamagata et al., 2009). As described previously, we identified the caudal part, or area F4, and the rostral part, or area F5, of the PMv (Matelli et al., 1985).

Muscle activity was recorded with stainless-steel wire electrodes (single stranded, $0.0762 \mathrm{~mm}$ in diameter) inserted percutaneously. The electromyographic activity was amplified, A-D converted, and stored. We monitored activities in the following muscles during task performance: trapezius, supraspinatus, infraspinatus, deltoid, biceps, triceps, flexor carpi radialis, and flexor carpi ulnaris, as well as the neck and paravertebral muscles.

\section{Behavioral tasks}

The monkeys were trained to perform three reaching-movement tasks described in our previous reports (Yamagata et al., 2009; Saga et al., 2013). Two monkeys (Monkeys 1 and 2) learned goal-directed tasks, that is, spatial goal, object goal, and a visually guided task (see Saga et al., 2013 for details), and two other monkeys (Monkeys 3 and 4) learned a symbolic cue task and a visually guided task (Yamagata et al., 2009). In the present study, we focused on the visually guided reaching task (VG task, Fig. 1A). For Monkeys 1 and 2 (TRN recording), after an inter-trial interval of 2000$2500 \mathrm{~ms}$, each trial began with the presentation of a fixation point (white square, visual angle of $1.4^{\circ}$ ) in the center of the screen (corresponding to T3 in Fig. 1B). When the monkey pressed the hold button with its right hand and gazed at the fixation point for 600 $1200 \mathrm{~ms}$, a blue-colored target in the shape of a circle or triangle was presented at one of five possible positions on the screen (Fig. 1B; visual angle of $7 \times 7^{\circ}$ for each target, $11^{\circ}$ between the centers of adjacent target positions). For Monkeys 3 and 4 (PMv recording), the target was colored gray, and the shape was square. When the color changed from blue or gray to white (the GO signal) after a delay of $1500-2500 \mathrm{~ms}$, the monkey made a reaching movement toward the target. The difference in VG tasks between recording from the PMv and from the TRN was that the target disappeared after presentation of the target (presentation period: $800 \mathrm{~ms}$ ) in PMv recording sessions. The target appeared again at the same position after a $1200-\mathrm{ms}$ delay period during PMv 

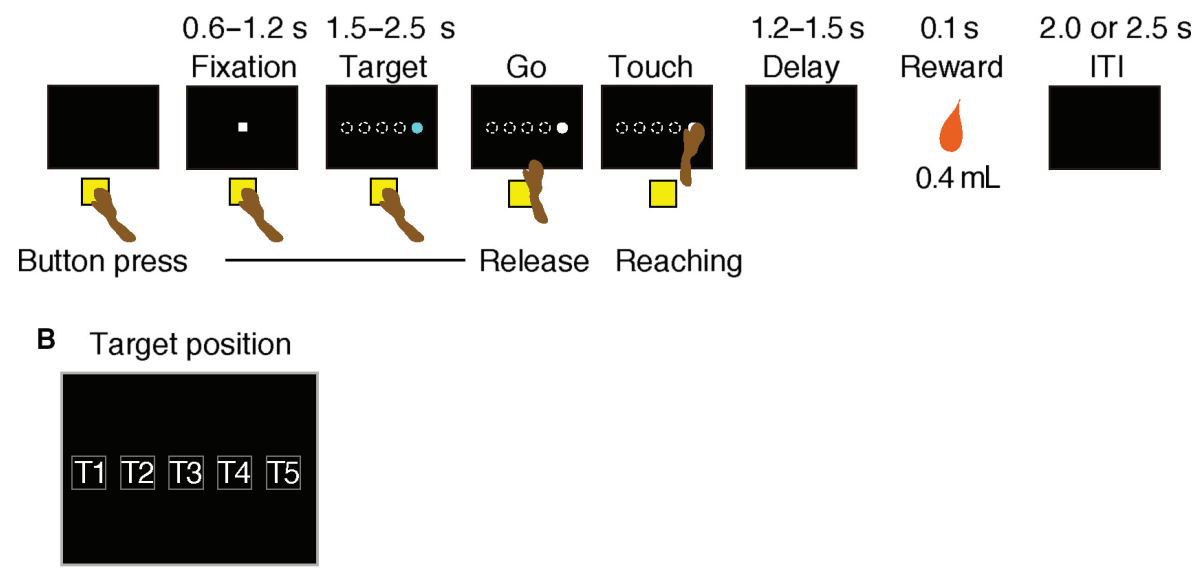

C
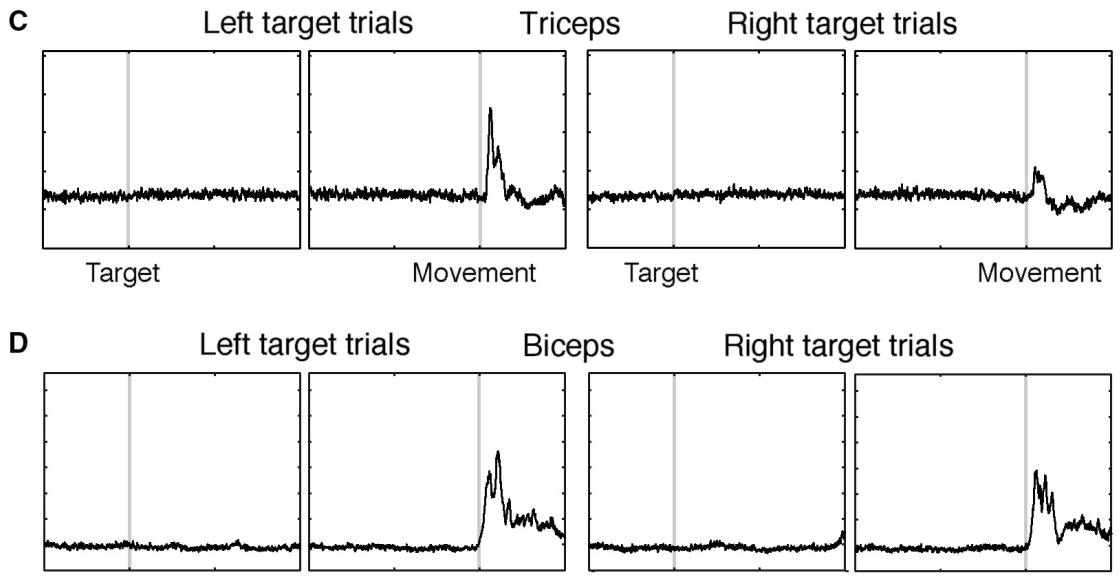

Target

Movement

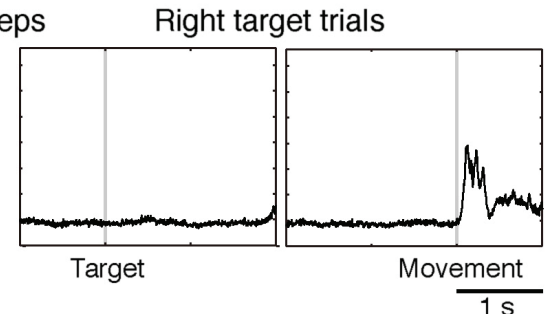

FIG. 1. Visually guided reaching task (VG task), target location, and muscle activity. (A) Temporal sequence of behavioral events. ITI, inter-trial interval. (B) Positions of targets on the screen were selected randomly from the five possible positions (T1-T5). The targets were triangular or circular in shape. (C) and (D) Electromyography (EMG) activity of the triceps (C) and biceps muscles (D) of the right arm. The EMGs were aligned with target appearance (left panel) and with movement onset (right panel) in the left and right target trials.

recording sessions (Fig. 1D in Yamagata et al., 2009). When the monkey touched the target, it disappeared immediately, and a drop of apple juice $(0.4 \mathrm{~mL})$ was delivered after $1200 \mathrm{~ms}$ or $1500 \mathrm{~ms}$ in TRN recordings and after $500 \mathrm{~ms}$ in PMv recordings. If the monkey released the button prematurely, that is, before the appearance of the GO signal, the target disappeared immediately, and an inter-trial interval with no reward began.

\section{Data analyses}

To examine visual-, set-, and movement-related activity without confounding by saccade-related activity, we attempted initially to find neurons related to saccadic eye movement. A saccadic eye movement was detected when the velocity of the eye movement was $\geq 40 \%$ and the gaze shift was $\geq 3^{\circ}$. Then, we compared neuronal activity during the $100-\mathrm{ms}$ period before saccade onset with that during the 100 -ms period after onset; activity from -3000 to $0 \mathrm{~ms}$ relative to target onset was analyzed separately for saccades in the four directions [upper right, upper left, lower right, and lower left, paired $t$-test with Bonferroni correction, $\alpha=0.0025$; (Nakayama et al., 2016)]. Neurons showing saccade-related activity were excluded from the neuronal database. Next, we analyzed neuronal activity during three task periods of the VG task: the posttarget period (50-250 ms after target presentation) to detect visualrelated activity; the pre-GO period (200 ms preceding presentation of the GO signal) to detect set-related activity; and the pre-touch period (200 ms preceding touching the target) to detect movementrelated activity. We compared the activity during the control period (200 ms before the onset of the visual signal) with that during each of the post-target, pre-GO, and pre-touch periods; this analysis was conducted separately for the left and right target positions (paired $t$ test with Bonferroni correction; $\alpha=0.01$, corrected). If the activity during the post-target, pre-GO, or pre-touch period was significantly different from that during the control period in either right or lefttarget trials, the neuron was defined as having visual-related, setrelated, or movement-related activity respectively.

To examine the spatial selectivity of neuronal activity, visualrelated, set-related, and movement-related activities were each analyzed using one-way ANOVA $(\alpha=0.05)$ with the main factor being target position (left: position 1 or 2 vs. right: position 4 or 5; Fig. 1B). We categorized the target position into two groups following our previous report (Yamagata et al., 2009). Based on this analysis, each response was classified into position-selective (POSITION $<0.05$ ) or non-selective activity (POSITION $\geq 0.05$ ). 


\section{Neuroanatomical study}

To identify the TRN subdivision interconnected with the PMv, we used retrograde transneuronal labeling with rabies virus in two monkeys (Monkeys 5 and 6: males, 6.9 and $6.0 \mathrm{~kg}$ ). The rabies virus (CVS-11 strain, $1.0 \times 10^{8}$ focus-forming units $/ \mathrm{mL}$ ) was from the Centers for Disease Control and Prevention (Atlanta, GA, USA) and was provided by Dr. S. Inoue (The National Institute of Infectious Diseases, Tokyo, Japan). For viral injections, we used the method described in our previous reports (Saga et al., 2011; Takahara et al., 2012). A viral suspension was injected slowly through a $10-\mu \mathrm{L}$ Hamilton microsyringe into the rostral and dorsal portions of the PMv (Fig. 2A and B). Along each injection track, viral deposits were placed at two different depths: 3 and $2 \mathrm{~mm}$ below the cortical surface. At each depth, $0.5 \mu \mathrm{L}$ of the viral suspension was deposited. Then, 3 days after the injections, the two monkeys (Monkeys 5 and 6) were anesthetized deeply with an overdose of sodium pentobarbital $(50 \mathrm{mg} / \mathrm{kg})$ and sacrificed by perfusion fixation with a mixture of $10 \%$ formalin and $15 \%$ saturated picric acid in $0.1 \mathrm{M}$ phosphate buffer (pH 7.4) (Saga et al., 2011; Takahara et al., 2012). This survival time permitted reaching the second stage of retrograde trans-synaptic transport of the virus, resulting in labeling of secondorder neurons that projected to the injection sites (PMv) disynaptically (Saga et al., 2011). Then, serial 50- or $60-\mu \mathrm{m}$-thick coronal sections were cut on a freezing microtome, and every sixth section was processed for immunohistochemical staining for rabies virus using a standard avidin-biotin-peroxidase complex method (see Saga et al., 2011). Following immersion in 1\% skimmed milk, the sections were incubated overnight with rabbit anti-rabies virus antibody (from Dr. S. Inoue) in $0.1 \mathrm{~m}$ phosphate-buffered saline (pH 7.4) containing $0.1 \%$ Triton $\mathrm{X}-100$ and $1 \%$ normal goat serum. The sections were then placed in the same fresh incubation medium containing biotinylated goat anti-rabbit IgG antibody $(1: 200$; Vector Laboratories), followed by the avidin-biotin-peroxidase complex kit (ABC Elite; Vector Laboratories). To visualize the antigen, the sections were reacted in $0.05 \mathrm{~m}$ Tris- $\mathrm{HCl}$ buffer $(\mathrm{pH}$ 7.6) containing $0.04 \%$ diaminobenzidine, $0.04 \%$ nickel chloride, and $0.002 \%$ hydrogen peroxide. The sections were mounted on gelatin-coated glass slides and examined by light microscopy (Eclipse 80i, Nikon, Tokyo, Japan).

\section{Histological analysis of a monkey used in the electrophysiological study}

After completing the electrophysiological recordings, perfusion fixation was conducted as described above in one monkey (Monkey 1). The perfused brain was removed from the skull, post-fixed in the same fresh fixative overnight at $4{ }^{\circ} \mathrm{C}$, and placed in $0.1 \mathrm{M}$ phosphate buffer ( $\mathrm{pH}$ 7.4) containing $30 \%$ sucrose. Serial $60-\mu \mathrm{m}$-thick coronal sections were then cut on a freezing microtome. The sections for verifying recording sites were mounted on gelatin-coated glass slides, stained with $1 \%$ cresyl violet, and then examined by light microscopy (Eclipse 80i, Nikon).

\section{Results}

\section{TRN subdivision connected with PMv across synapses}

The TRN does not send direct projections to the cerebral cortex. This thalamic nucleus is considered to project to cortical areas via the thalamic nuclei. In the cortex, we focused on the PMv, which has been shown to play a central role in reaching movements. To identify the TRN sector projecting to the PMv, we injected rabies virus in to the PMv (Fig. 2A and B). By 3 days after the rabies injections, second-order neuron labeling had occurred. Some TRN neurons were labeled with the virus. Dense clusters of labeled neurons were found in the rostro-dorsal sector of the TRN (TRNrd; Fig. 2C and D, D'), and also mainly in the ventral thalamic nuclei. In the thalamic nuclei, dense labeled neurons (the first or secondorder ones) were observed in the nucleus $\mathrm{X}$ (Fig. $2 \mathrm{C}$ and D), and less densely in the ventroanterior nucleus (VA), the oral division of the ventrolateral nucleus (VLo), the caudal division of the ventrolateral nucleus (VLc), as well as in the mediodorsal nucleus (MD). The TRN neurons were considered to project to the PMv via these thalamic relay nuclei. The results indicate that the TRNrd is part of the multisynaptic network centered on the PMv, and that this portion of the TRN sector may be involved specifically in reaching movements.

\section{Task performance and muscle activity}

The monkeys performed the VG task at a success rate of $>98 \%$, executing reaching movements promptly after the onset of the GO signal. The reaction time (RT) and movement time (MT) were $<600 \mathrm{~ms}$ in all monkeys (Table 1). Figure $1 \mathrm{C}$ and D show the activity of the triceps and biceps muscles in Monkey 1. As in these examples, the muscles in the right forelimb had movement-related activity changes only during reaching movements. Thus, the monkeys made a movement only during the task performance, while in the remaining periods they sat quietly.

\section{Neuronal recordings from the TRNrd}

We examined neuronal activity of the TRN in the left hemisphere, the side opposite the task-performing right arm. Recordings were carried out from the TRNrd (Fig. 2E), which had been identified as projecting to the PMv through thalamic neurons. The electrode was advanced vertically in the dorsoventral direction. Before contacting neurons in the TRNrd, the electrode went through sporadically firing striatal (caudate) cells and passed the white matter (Fig. 2E). In addition to their spatial localization, TRNrd neurons showed high spontaneous discharge rates. During the 500-ms period before the presentation of the fixation point, the discharge rate of the TRNrd neurons was $40 \pm 2$ spikes/s $(n=106$; mean \pm SEM $)$. This value was higher than that of PMv neurons $(n=443 ; 10 \pm 1$ spikes/s, mean \pm SEM, $P<0.001$, two-tailed $t$-test). The higher discharge rates of the TRNrd neurons are consistent with those in the visual sector of the TRN (McAlonan et al., 2008). Subsequently, at the transition from the TRNrd to the thalamic relay nuclei and white matter, the neuronal discharge rate dropped, and the cellular activity disappeared respectively. In one monkey, the recording sites were verified histologically with reference to markings made by passing anodal direct currents (20$40 \mu \mathrm{A}$ for $20 \mathrm{~s}$ ) through the recording electrodes (Nomoto et al., 2010) (Fig. 2E, left and middle panels) and the gliosis along the electrode track (Fig. 2E, right panel). The recording sites were confirmed in accordance with those estimated based on the magnetic resonance images. Although we recorded neuronal activity from the thalamic relay nuclei, we did not sample enough data from the nucleus $\mathrm{X}$ that is the main relay nucleus to the PMv. Therefore, we did not analyze neurons in the thalamic relay nuclei.

We examined the impact of saccadic eye movement on activity in each neuron (see Materials and Methods). Of the 106 TRNrd neurons, none showed significant activity modulation in relation to saccadic eye movement (paired $t$-test with Bonferroni correction, 


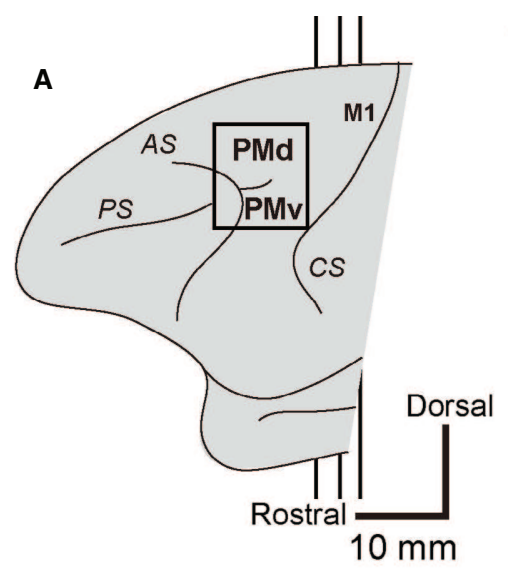

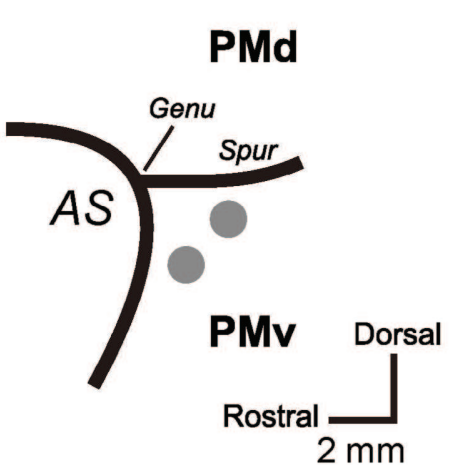

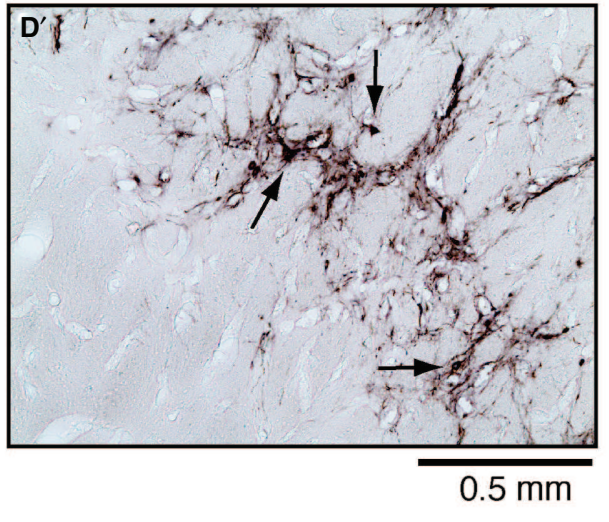

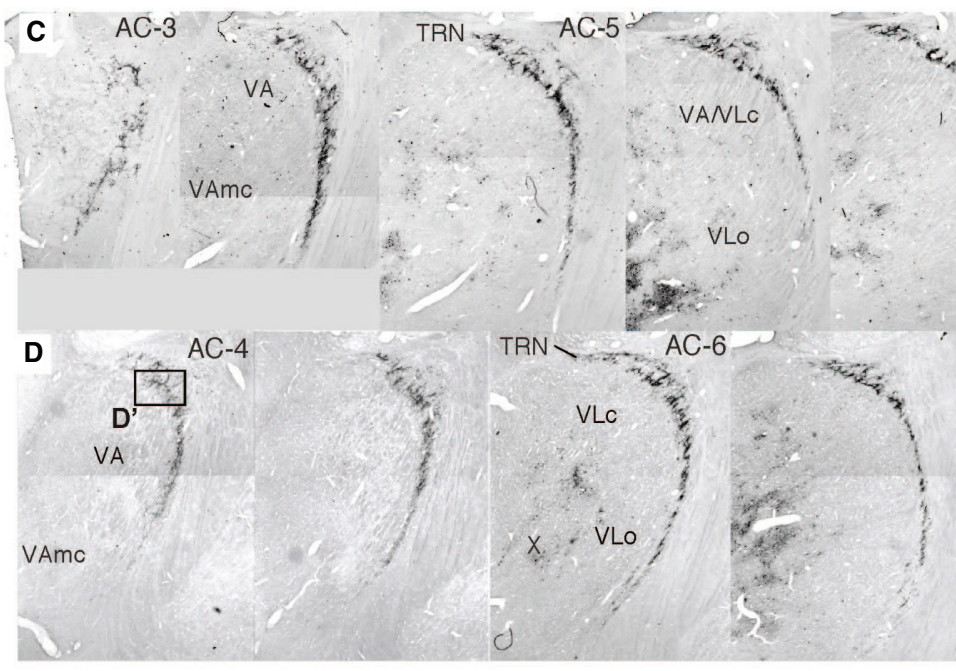

E
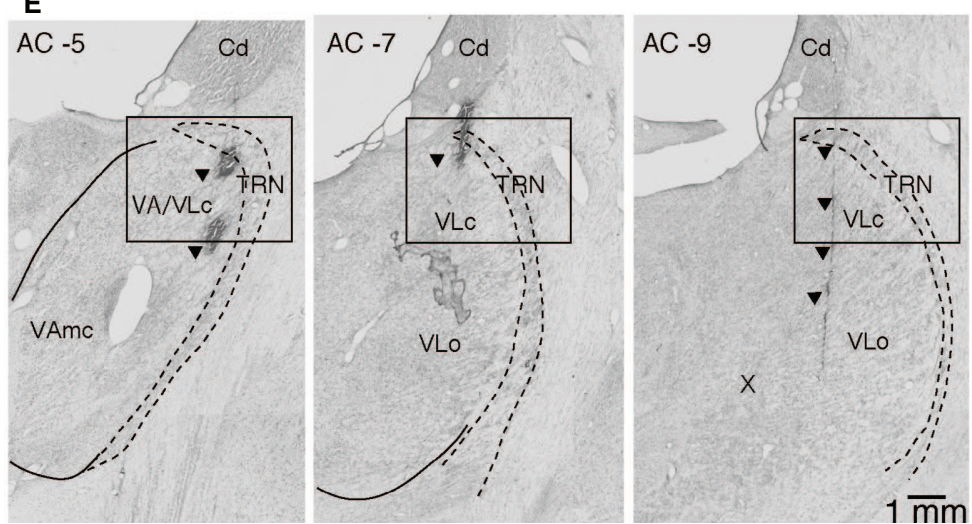

F

AC 4 to AC -6

AC -7 to $A C-8$

AC -9 to $A C-11$
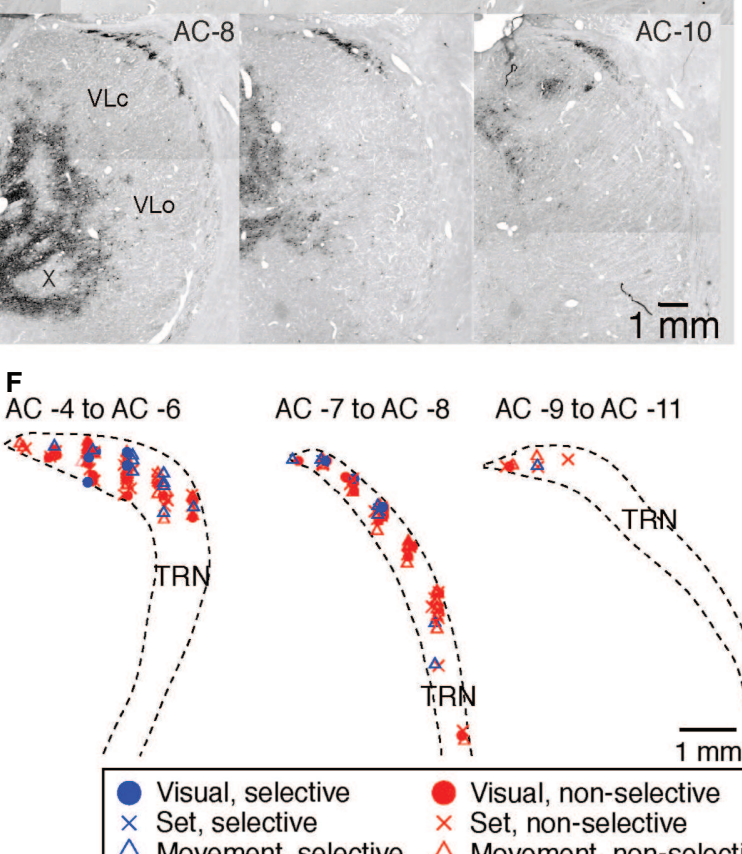

$1 \overline{\mathrm{m} m}$

FIG. 2. Sites of rabies injections in the PMv, distribution of rabies-labeled neurons in TRN, histological reconstructions of recording sites, and locations of visual-, set-, and movement-related neurons. (A) Lateral view of the monkey brain. The rectangular area is enlarged in B. (B) Rabies injection sites. The two filled circles indicate the injection sites. (C) Rabies-labeled neurons (in black) in the TRN and motor thalamic nuclei (VA, X, VLo, VLc) in Monkey 5. Photomicrographs of coronal sections at distinct rostrocaudal levels are arranged from left (rostral) to right (caudal). (D) Photomicrographs of coronal sections through the TRN at distinct rostrocaudal levels in Monkey 6. The small rectangle showing infected neurons is enlarged in $D^{\prime}$. (E) Triangles indicate sites marked by passing an electrical current (left and middle panels) and gliosis along the electrode track (right panel). (F) The three rectangular areas demarcated in $\mathrm{E}$ are enlarged to show the locations of the neurons. Visual- $(\mathbf{O})$, set- $(\times)$, and movement-related $(\Delta)$ neurons are intermingled within the TRN. Blue, directionselective neurons; red, direction-non-selective neurons. Each drawing represents an overlay of three coronal sections at distinct rostrocaudal levels. AS, arcuate sulcus; Cd, caudate nucleus; CS, central sulcus; Genu, genu of the AS; M1, primary motor cortex; PMd, dorsal premotor cortex; PS, principal sulcus; Spur, spur of the AS; VA, ventroanterior nucleus; VLo, oral division of ventrolateral nucleus; VLc, caudal division of ventrolateral nucleus.

$P>0.05$, corrected). In the PMv, of the 498 neurons, $55(11 \%)$ showed saccade-related activity (paired $t$-test with Bonferroni correction, $P<0.05$, corrected). We excluded those PMv neurons from subsequent analyses. Thus, in this study, we analyzed 106 TRNrd neurons and $443 \mathrm{PMv}$ neurons that had no apparent direct relationship with saccadic eye movement. 
TABLE 1. Reaction and movement times

\begin{tabular}{lll}
\hline & \multicolumn{2}{l}{ Target position } \\
\cline { 2 - 3 } & Left & Right \\
\hline $\begin{array}{l}\text { Monkey 1 } \\
\quad \text { Reaction time } \\
\quad \text { Movement time }\end{array}$ & $366 \pm 104$ & \\
$\begin{array}{l}\text { Monkey 2 } \\
\quad \text { Reaction time } \\
\text { Movement time }\end{array}$ & $348 \pm 118$ & $353 \pm 99$ \\
$\begin{array}{l}\text { Monkey 3 } \\
\text { Reaction time } \\
\quad \text { Movement time }\end{array}$ & $351 \pm 112$ & $349 \pm 143$ \\
$\begin{array}{l}\text { Monkey 4 } \\
\text { Reaction time } \\
\text { Movement time }\end{array}$ & $402 \pm 156$ & $335 \pm 97$ \\
\hline
\end{tabular}

Times are expressed as means \pm standard deviations (ms).

\section{Visual-, set-, and movement-related neurons in the TRNrd}

Figure 3 shows four TRNrd neurons during the VG task. Generally, TRNrd neurons showed visual-related activity in response to the onset of visual signals (Fig. 3A, B and D), set-related activity during preparation for reaching movement (Fig. 3B and C), and movementrelated activity during execution of the movement (Fig. 3A-D).

Among the 106 TRNrd neurons, 91 (86\%) exhibited activity modulation in at least one of the three task phases. To gain insight into overall activity modulation, population activity in the 91 taskrelated neurons was assessed separately for the left- and right-side trials (Fig. 4). This indicated that neuronal activity was modulated in response to visual signal onset, during the preparation, and execution of reaching movements. The overall activity was increased and was similar to the right- and left-target trials.

By analyzing the activity of each neuron, we found that 43 (41\%), 45 (42\%), and 64 (60\%) neurons displayed visual-, set-, and movement-related activity respectively (paired $t$-test with Bonferroni correction, $\alpha=0.05$, corrected). Of these, 50 (55\%) neurons exhibited activity modulation in two or three task phases. Thirteen neurons (14\%) showed visual- and set-related activity, $13(14 \%)$ demonstrated visual- and movement-related activities, and 13 (14\%) showed set- and movement-related activity. Additionally, 11 (12\%) neurons displayed visual-, set-, and movement-related activity. We then compared the baseline activities of single phase and multiple phase neurons, and found that their baseline activities were not different (single phase neurons, $44.6 \pm 3.0$ spike/s, $n=41$;
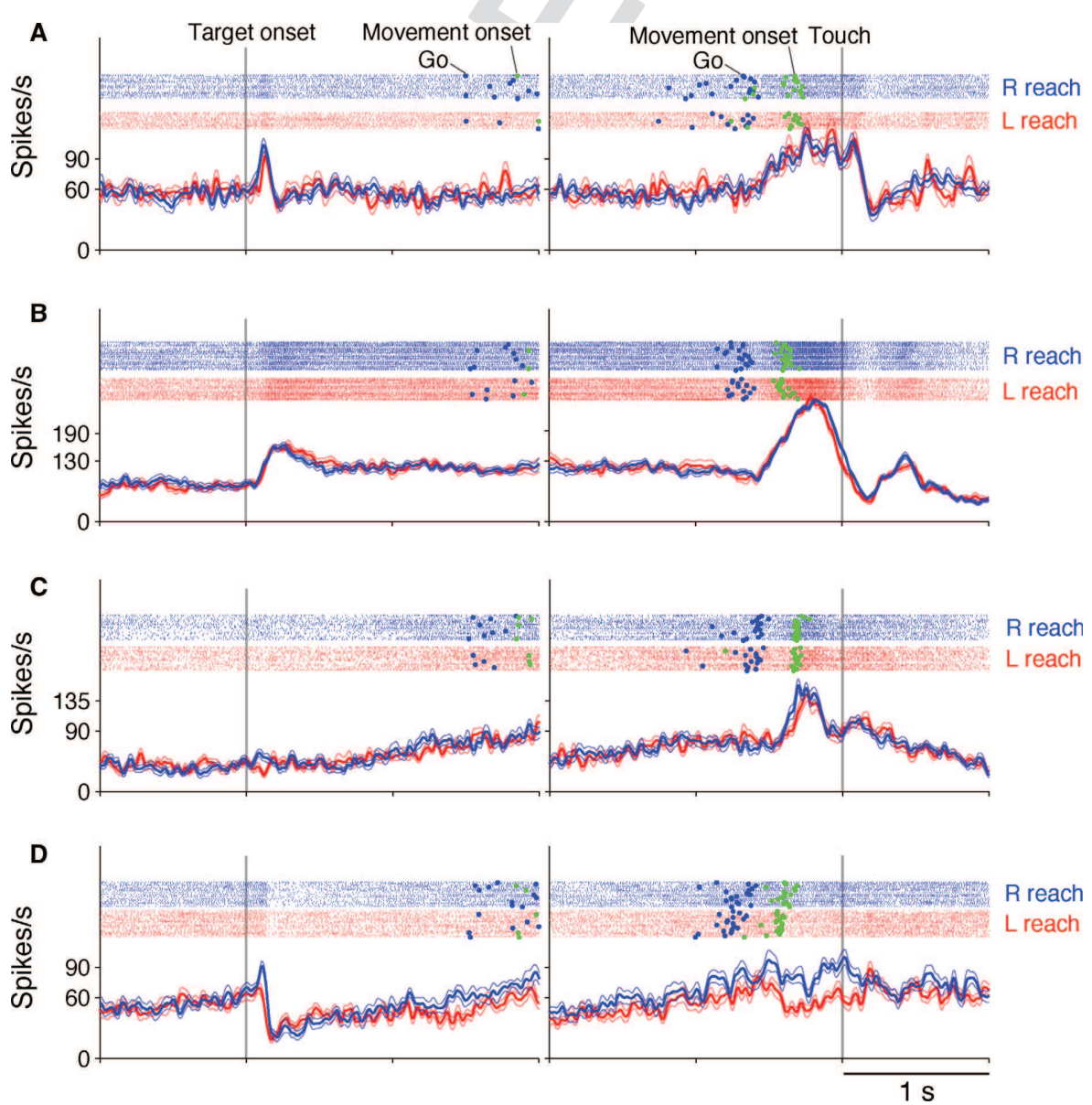

FIG. 3. Four examples of TRN neurons. (A) A neuron showing spatially non-selective visual- and movement-related activity. (B) A neuron with spatially non-selective visual-, set-, and movement-related activity. (C) A neuron showing spatially non-selective set- and movement-related activity. (D) A neuron showing spatially selective visual- and movement-related activity. The rasters and spike density functions (smoothed using a Gaussian kernel, $\sigma=10$ ms; mean \pm SEM) in blue indicate trials in which targets were presented in the right-hand position, and those in red indicate trials in which targets were presented in the left-hand position. Left panels are aligned with the onset of the visual target, and right panels are aligned with the target touch. Blue dots in the raster indicate the onset time of the GO signal. Green dots in the raster indicate movement onset. 

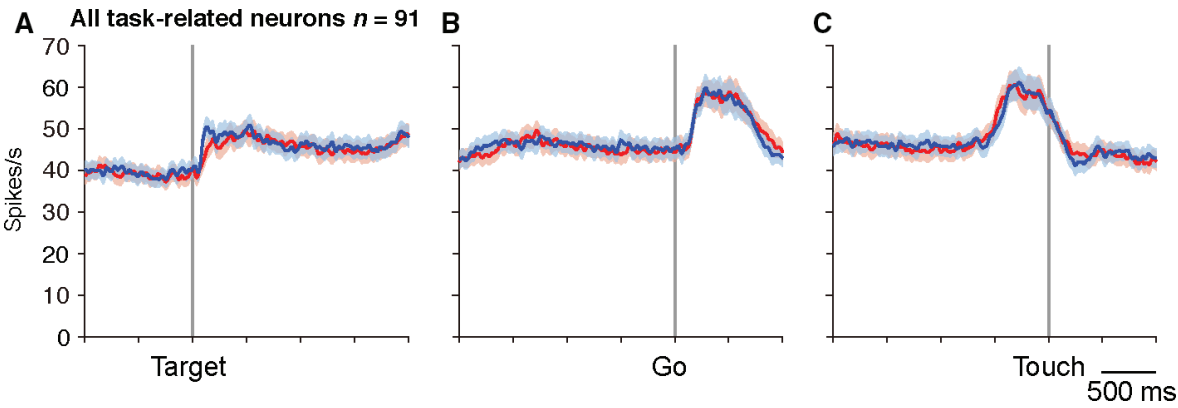

FIG. 4. Population activity of all task-related TRNrd neurons $(n=91)$. (A-C) Two sets of population activity (mean \pm SEM; red, left target-trials; blue, right target trials) are aligned with target onset (A), GO signal onset (B), and target touch (C).

multiphasic neurons, $38.6 \pm 3.0$ spike/s, $n=50 ; P=0.1728$, twotailed $t$-test).

From the PMv, we sampled 443 neurons having no eye-movement effect. Of these, 375 neurons showed modulation in the VG task: $164(44 \%), 292$ (78\%), and $300(80 \%)$ neurons showed visual-, set-, and movement-related activity respectively. In the following sections, we compare the properties of these activities between the TRNrd and the PMv.

\section{Visual-related neurons in the TRNrd and the PMv}

We first analyzed the properties of the 43 visual-related TRNrd neurons (Fig. 1A). The two neurons shown in Figs 3A and B exhibited excitatory phasic responses after the appearance of the visual targets. The activity was similar to the right- and left target-positions $(P>0.05$, two-sample $t$-test). The neuron in Fig. 3D showed visualrelated phasic responses, and the activity was greater in the right than in the left-target position $(P<0.05$, two-sample $t$-test). Activity in $35(81 \%)$ neurons did not differ between the right and left-target positions, whereas in eight neurons, it did. In the PMv, 75 (46\%) of visual-related neurons were not selective for the target position (Fig. 5D), whereas the remaining 89 neurons were. Thus, TRNrd neurons were more frequently non-selective for the target position than PMv neurons were (Table 2, $P<0.0001$, Fisher's exact test).

We classified the spatially non-selective activity into activityincrease and activity-decrease types with respect to baseline activity (the 200-ms period before target appearance). In the TRNrd, 91\% (32/ 35 ) of neurons were classified as the activity-increase type. In the PMv, 73\% (55/75) of neurons were the activity-increase type. Thus, the fraction of neurons with activity increase was greater in the TRNrd than the PMv ( $P=0.04243$, Fisher's exact test). Figure 5A shows the population activity of TRNrd neurons with activity increase $(n=32$, blue for right target and red for left target) and with activity decrease $(n=3$, cyan for right target and orange for left target). Figure 5D shows the population activity of PMv neurons with activity increase $(n=55)$ and with activity decrease $(n=20)$. At the population level, the latencies of spatially non-selective TRNrd neurons with activity increase were $104 \mathrm{~ms}$ for the right target and $111 \mathrm{~ms}$ for the left target. This timing did not differ when evaluated at the single-neuron level (Table 3, $P=0.6569$, two-tailed paired $t$-test). The latencies of spatially non-selective $\mathrm{PMv}$ neurons with activity increase were $105 \mathrm{~ms}$ for the right position and $94 \mathrm{~ms}$ for the left position at the population level (Table 3, $P=0.9508$, two-tailed $t$-test, evaluated at the single-neuron level). The timing did not differ between the TRNrd and the PMv $(P=0.2686$ for right target, $P=0.4069$ for left target; two-tailed $t$-test, evaluated at the single-neuron level). The population activities of TRNrd neurons with activity increases reached peaks at
$141 \mathrm{~ms}$ for the right target and $142 \mathrm{~ms}$ for the left target. The population activities of PMv neurons with activity increases reached peaks at $140 \mathrm{~ms}$ for the right target and $162 \mathrm{~ms}$ for the left target (Fig. 5A and D). The peak timing was slightly earlier in PMv than in TRNrd for the right target $(P=0.0215$, evaluated at the single-neuron level, twotailed $t$-test), whereas the two did not differ for the left target $(P=0.1263$, two-tailed $t$-test). These results revealed that the spatially non-selective activity increase in TRNrd and PMv neurons began and developed with similar timing, although the timing for PMv neurons tended to be earlier.

Next, the activity decrease in the spatially non-selective neurons was analyzed (Fig. 5A and D). This suggested that the activity decrease occurred via distinct neural mechanisms in the TRNrd and PMv. The PMv neurons showed a build-up activity preceding the appearance of the visual signals, and this activity was suppressed in response to the appearance of the visual targets. In contrast, the activity decrease of the TRNrd neurons was due to reduced baseline activity at higher discharge rates $(\sim 40 \mathrm{~Hz})$.

Spatially selective visual-related neurons were also analyzed ( $n=8$ for TRNrd, $n=89$ for PMv). Out of eight neurons, six (75\%) exhibited multiphasic response (set-related activity, $n=2$; movement-related activity, $n=2$; set- and movement-related activity, $n=2$ ). The population activity of spatially selective visualrelated neurons in the TRNrd and PMv revealed that phasic activity was observed only when the target appeared in the preferred positions (Fig. 6A and E). The latency of TRNrd neurons' response to the preferred position was $99 \mathrm{~ms}$. The peak timing of spatially selective visually related neurons did not differ from that of spatially non-selective neurons $(P=0.6136$, two-tailed $t$-test, evaluated at the single-neuron level). The latency of the PMv neuron population for the preferred position was $86 \mathrm{~ms}$, which did not differ from that of TRNrd neurons $(P=0.9417$, two-sample $t$-test, evaluated at the single-neuron level). The population activity in response to the preferred target reached peaks at $137 \mathrm{~ms}$ in the TRNrd and $108 \mathrm{~ms}$ in the PMv (Fig. 6A and E, $P=0.6807$, two-tailed $t$-test). In terms of spatial selectivity, the TRNrd reached peak selectivity at $132 \mathrm{~ms}$ after the target onset, whereas the PMv did so at $107 \mathrm{~ms}$ after target onset (Fig. 6C and G). The peak timing of the TRNrd and PMv did not differ $(P=0.1086$, two-tailed $t$-test, evaluated at the single-neuron level). These results showed that spatial selectivity began at similar times in the TRNrd and PMv, whereas the development of selectivity tended to be earlier in the PMv than in the TRNrd.

\section{Set-related neurons in the TRNrd and PMv}

Set-related activity during preparation for a reaching movement was also observed. Figure $3 \mathrm{C}$ shows a TRNrd neuron with set-related 


\section{A TRN non-selective neurons}

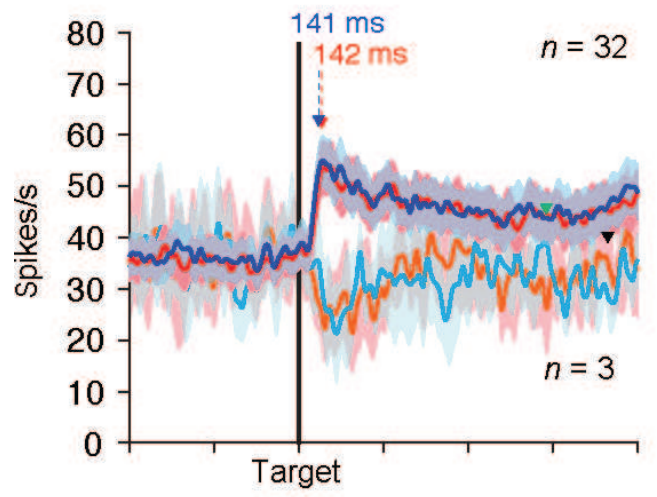

PMv non-selective neurons

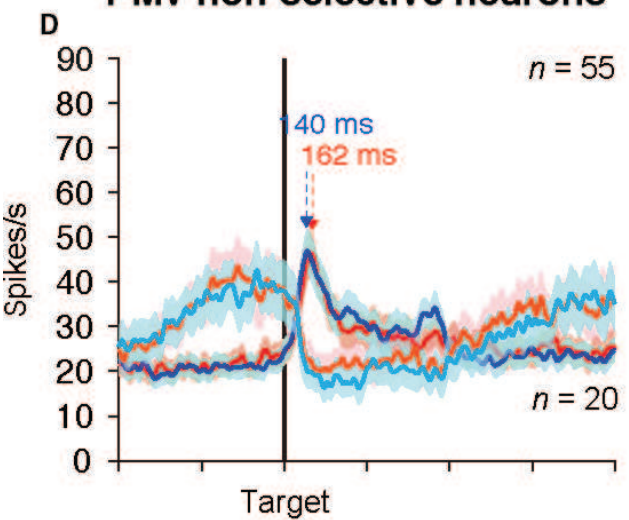

B

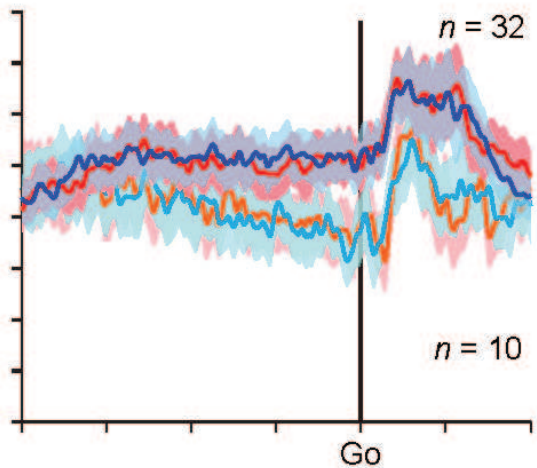

E

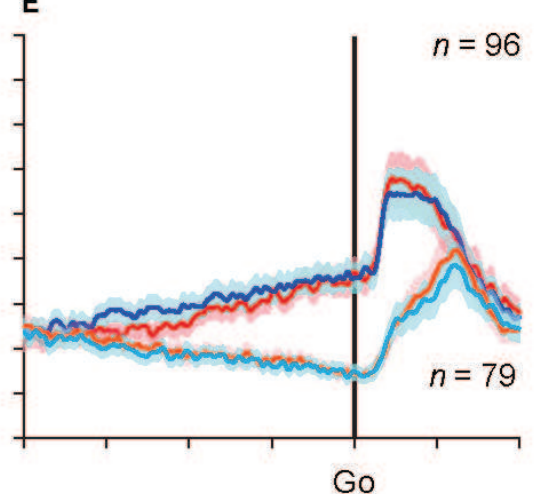

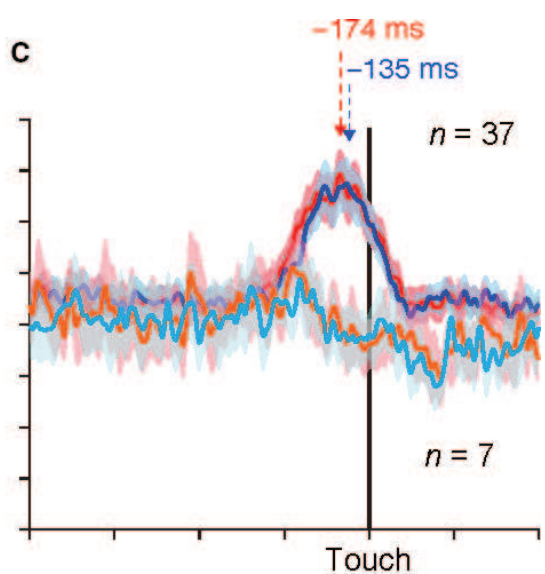

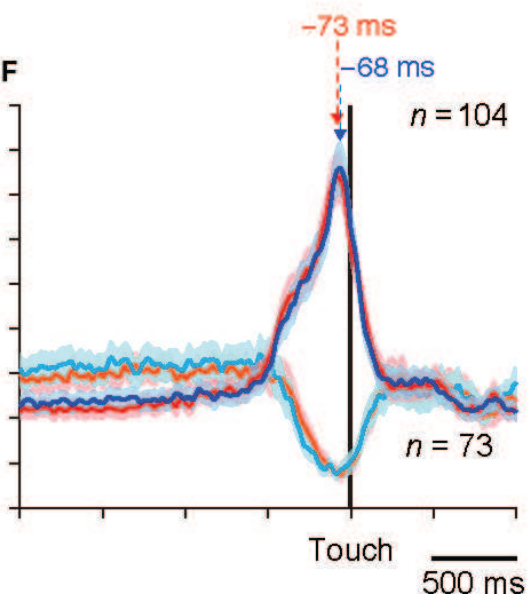

FIG. 5. Population activity of spatially non-selective neurons in TRNrd (A-C) and PMv (D-F). (A) Population activity (mean \pm SEM) of visual-related TRNrd neurons with activity increases ( $n=32$; blue, right target trials; red, left-target trials) and activity decreases ( $n=3$; cyan, right target trials; orange, left-target trials). Activity is aligned with the onset of visual targets. (B) Population activity of set-related TRNrd neurons with activity increases $(n=32)$ and activity decreases $(n=10)$. Activity is aligned with GO onset. (C) Population activity of movement-related neurons with activity increases $(n=37)$ and activity decreases $(n=7)$. (D) Population activity of visual-related PMv neurons with activity increases $(n=55$; blue, right target trials; red, left-target trials) and decreases $(n=20$; cyan, right target trials; orange, left-target trials). (E) Set-related population activity of PMv neurons with activity increases $(n=96)$ and decreases $(n=79)$. (F) Movement-related population activity of PMv neurons with activity increases $(n=104)$ and decreases $(n=73)$. Activity is aligned with target touch. Blue and red inverted triangles in A, C, D, F, indicate peak or trough activity (blue, right target position; red, left-target position). The ordinates indicate instantaneous discharge rates in spikes $/ \mathrm{s}$. Scale bar $=500 \mathrm{~ms}$.

TABLE 2. Summary of the fraction of spatially non-selective neurons and the ratio of neurons with excitatory responses

\begin{tabular}{lll}
\hline & $\begin{array}{l}\text { Spatially non-selective } \\
\text { neurons }\end{array}$ & $\begin{array}{l}\text { Neurons with } \\
\text { excitatory responses }\end{array}$ \\
\hline $\begin{array}{c}\text { Visual-related } \\
\text { neurons }\end{array}$ & TRN $(81 \%) \gg \mathrm{PMv}(46 \%)$ & $\mathrm{TRN}(91 \%) \gg \mathrm{PMv}(73 \%)$ \\
$\begin{array}{c}\text { Set-related } \\
\text { neurons }\end{array}$ & $\mathrm{TRN}(93 \%) \gg \mathrm{PMv}(60 \%)$ & $\mathrm{TRN}(76 \%) \gg \mathrm{PMv}(55 \%)$ \\
$\begin{array}{c}\text { Movement-related } \\
\text { neurons }\end{array}$ & $\operatorname{TRN~}(69 \%)>\mathrm{PMv}(59 \%)$ & $\mathrm{TRN}(84 \%) \gg \mathrm{PMv}(59 \%)$ \\
\hline
\end{tabular}

〉, statistically significant $(P<0.05)$; $>$, statistically non-significant although the fraction is greater $(P \geq 0.05)$.

activity. Activity in this neuron built up in preparation for the reaching movement. Figure 3B shows another TRNrd neuron with setrelated activity. This neuron showed a sustained activity increase during the preparation period. The set-related activity of these neurons was similar for the right- and left-reach targets. In the TRNrd,
TABLE 3. Timing of activity modulation of visual-related neurons in TRN and PMv (criterion $=25 \%$ of peak)

\begin{tabular}{llr}
\hline & TRN & PMv \\
\hline Spatially non-selective & Mean \pm SEM & \\
$\quad$ Right target & $104(77 \pm 12)$ & $94(60 \pm 8)$ \\
$\quad$ Left target & $111(70 \pm 9)$ & $105(61 \pm 6)$ \\
Spatially selective & $99(75 \pm 32)$ & $86(72 \pm 12)$ \\
$\quad$ Preferred target & $192(106 \pm 57)$ & $72(69 \pm 8)$ \\
$\quad$ Non-preferred target & & \\
\hline
\end{tabular}

Numbers indicate the timings of population activity. Numbers in parentheses indicate means \pm standard error of means $(\mathrm{ms})$ of single neurons.

42 of the $45(93 \%)$ set-related neurons were spatially non-selective for the direction of reaching movement. The remaining three neurons were spatially selective and showed multiphasic responses (visual-related activity, $n=1$; movement-related activity, $n=1$; visual- and movement-related activity, $n=1$ ). In the PMv, 175 of the $292(60 \%)$ neurons were of the spatially non-selective type. 

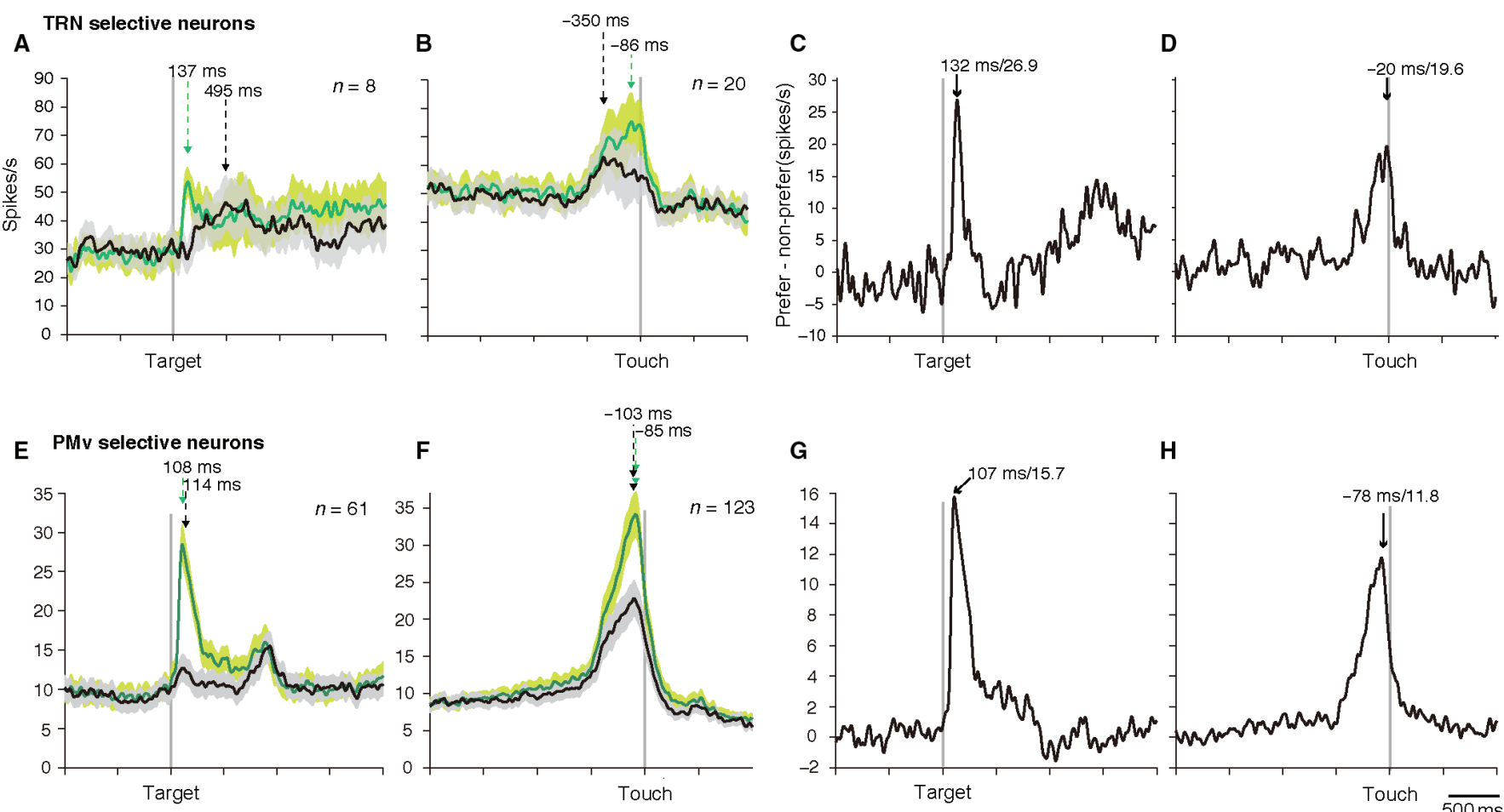

$\mathrm{H}$

FIG. 6. Population activity of spatially selective neurons. (A) Population activity (mean \pm SEM) of visual-related TRNrd neurons for preferred (green) and non-preferred (black) positions. Activity is aligned with target onset. (B) Population activity (mean \pm SEM) of movement-related TRNrd neurons. Activity is aligned with target touch. Arrows indicate the timing of activity peaks (green, preferred positions; black, non-preferred positions). (C) and (D) Spatial selectivity between the preferred and non-preferred target positions of the spatially selective TRNrd neurons (C, visual-related neurons; D, movement-related neurons). Arrows indicate the timing of peak of selectivity (green, preferred positions; black, non-preferred positions). E-H. Population activity (E and F) and population selectivity activity $(\mathrm{G}$ and $\mathrm{H}$ ) of PMv neurons are shown in the same formats as for the TRNrd neurons (A-D). In A-H, the ordinates indicate instantaneous selectivity in spikes/s. Scale bar $=500 \mathrm{~ms}$.

Thus, the proportion of spatially non-selective neurons was greater in the TRNrd than in the PMv $(P<0.0001$, Fisher's exact test). In the TRNrd, a majority $(n=32 ; 76 \%)$ of the spatially non-selective neurons showed an activity increase, whereas the remaining 10 (24\%) showed an activity decrease. In the PMv, non-selective neurons showed decreased $(n=79,45 \%)$ and increased activity $(n=96,55 \%$; Fig. 5E). The fraction of neurons with activity increase was greater in the TRNrd than in the PMv (Table 2, $P=0.0003$, Fisher's exact test). These results showed that during preparation for movement, direction selectivity was lower in the TRNrd than in the PMv and that the TRNrd neurons were characterized by an activity increase.

\section{Movement-related neurons in TRNrd and PMv}

The TRNrd neurons in Figs $3 \mathrm{~A}-\mathrm{C}$ showed a robust increase in movement-related activity. Activity in these neurons was similar in the right- and left-target positions $(P=0.4923$, two-sample $t$-test $)$. In the TRNrd, of the 64 movement-related neurons, 20 (31\%) were spatially selective for the target position (Fig. 3B and C, $P=0.00369$, binomial test). Out of these, 11 (55\%) neurons showed multiphasic responses (visual-related activity, $n=5$; setrelated activity, $n=3$; visual- and set-related activity, $n=3$ ). In the PMv, 123 of 300 (41\%) neurons were selective for the target position. In contrast to the visual- and set-related neurons, the fraction of spatially selective neurons did not differ between the TRNrd and the PMv $(P=0.1606$, Fisher's exact test $)$.

Of the 44 spatially non-selective TRNrd neurons, 37 (84\%) showed an activity increase, and the remaining $7(16 \%)$ displayed an activity decrease. In the PMv, 104 spatially non-selective neurons (59\%) exhibited an activity increase, and 73 (41\%) showed an activity decrease. The fraction of those showing an increase in the TRNrd was significantly greater than that in the PMv (Table 2, $P=0.0015$, Fisher's exact test). Thus, the spatially non-selective movement-related TRNrd neurons, like non-selective visual- and set-related neurons, were characterized by activity increases. Figure 5C (blue and red) shows the population activity of the movement-related TRNrd neurons with activity increases, and Fig. 5C (cyan and orange) shows the population activity of those with activity decreases. The lead times of population activity of the TRNrd neurons with activity increases were $-496 \mathrm{~ms}$ and $-514 \mathrm{~ms}$ relative to touching the right and left targets, respectively, and those of the PMv neurons were $-457 \mathrm{~ms}$ (right target) and $-488 \mathrm{~ms}$ (left target, Table 4) respectively. The lead times of activity in the TRNrd and PMv neurons at the single-neuron level did not differ for either position ( $P>0.05$, two-tailed $t$-test). The activities of TRNrd neurons peaked at $-135 \mathrm{~ms}(67.4 \mathrm{spikes} / \mathrm{s})$ and $-174 \mathrm{~ms}(69.2$ spikes/s) relative to touching the right and left targets respectively. The activities of $\mathrm{PMv}$ neurons peaked at $-73 \mathrm{~ms}$ and $-68 \mathrm{~ms}$ relative to touching the right and left targets respectively (Fig. $5 \mathrm{C}$ and F). The peak timing in the TRNrd and PMv $(P=0.3882$ for the right target, $P=0.9521$ for the left target, two-tailed $t$-test, evaluated at the single-neuron level) did not differ. Because the overall movement time was $<400 \mathrm{~ms}$ (Table 1), these results showed that the movementrelated activity of spatially non-selective TRNrd neurons, as well as that of the PMv neurons, began earlier than the initiation of movement. They also revealed that the timing of activity modulation of TRNrd neurons was similar to that of PMv neurons. 
TABLE 4. Lead time, timing of activity peak, and peak firing rates of spatially non-selective movement-related neurons in the TRNrd and PMv

\begin{tabular}{|c|c|c|c|c|c|c|}
\hline \multirow[b]{2}{*}{ Target type } & \multicolumn{3}{|l|}{ Movement onset } & \multicolumn{3}{|l|}{ Touch } \\
\hline & Lead time (ms) & $\begin{array}{l}\text { Timing of } \\
\text { activity } \\
\text { peak (ms) }\end{array}$ & $\begin{array}{l}\text { Peak } \\
\text { firing rate } \\
\text { (spikes/s) }\end{array}$ & Lead time (ms) & $\begin{array}{l}\text { Timing of } \\
\text { activity } \\
\text { peak (ms) }\end{array}$ & $\begin{array}{l}\text { Peak } \\
\text { firing rate } \\
\text { (spikes/s) }\end{array}$ \\
\hline \multicolumn{7}{|l|}{ TRN } \\
\hline Right & $-121(-54 \pm 67)$ & 66 & 67.8 & $-496(-407 \pm 22)$ & -135 & 67.4 \\
\hline Left & $-165(-154 \pm 49)$ & 219 & 68.1 & $-514(-430 \pm 23)$ & -174 & 69.2 \\
\hline \multicolumn{7}{|l|}{$\mathrm{PMv}$} \\
\hline Right & $-197(-73 \pm 19)$ & 154 & 38.9 & $-457(-358 \pm 12)$ & -73 & 38.9 \\
\hline Left & $-196(-45 \pm 19)$ & 143 & 38.8 & $-488(-383 \pm 11)$ & -58 & 38.1 \\
\hline
\end{tabular}

Numbers indicate the timings of population activity (ms) and the peak firing rate (spikes/s). Numbers in parentheses indicate means \pm standard error of means (ms) of single neurons.

Next, we focused on the direction-selective neurons. The movement-related activity of the TRNrd neuron shown in Fig. 3D was greater for the right than for the left target. With respect to touching the target, activity modulation of the population activity began at $-444 \mathrm{~ms}$ in the TRNrd and $-455 \mathrm{~ms}$ in the PMv in the case of the preferred target location (Table 5). At the single-neuron level, PMv neurons started discharging earlier than TRNrd neurons did (twotailed $t$-test, $P=0.0001$; Table 5). The population activity in the TRNrd reached a peak at $-86 \mathrm{~ms}$ relative to the target touch in the preferred location, whereas the peak in the case of the PMv was at $-85 \mathrm{~ms}$. The greatest activity difference between the preferred and the non-preferred locations was observed at $-20 \mathrm{~ms}$ (19.6 spikes/s) in the TRNrd and at $-78 \mathrm{~ms}$ (11.8 spikes/s) in the PMv relative to target touch (Fig. 6D and H). The timing of the greatest differences did not differ between the TRNrd and the PMv $(P=0.9982$, twotailed $t$-test, evaluated at the single-neuron level). These results revealed that the direction selectivity of movement-related neurons began later in the TRNrd than in the PMv, although the subsequent development of the direction selectivity was similar in the two regions.

\section{Locations of the visual-, set-, and movement-related neurons within the TRNrd}

The neurons recorded were located within the TRNrd (Fig. 2E, F), corresponding to the portion in which neurons project to the PMv in a disynaptic manner (Fig. 2C and D). Neurons with visual- (-), set$(\times)$, and movement-related activity $(\Delta)$ were distributed widely in the sampled TRN areas (Fig. 2F).

\section{Discussion}

We analyzed the roles of TRN neurons in visually guided reaching movements. First, we used retrograde transneuronal labeling with rabies virus, demonstrating that the TRNrd projects disynaptically to the PMv, which plays a central role in reaching movements. Next, we recorded the activities of neurons in the TRNrd and PMv while monkeys performed a visually guided reaching task. The TRNrd and PMv neurons showed visual-, set-, and movement-related activity modulation, indicating that the TRNrd and PMv are involved in the reception of visual signals and in the preparation for and execution of movement. The fraction of neurons that were non-selective for the location of visual signals and the direction of reaching movement was greater in the TRN than in the PMv. Furthermore, the fraction of neurons with activity increases was greater in the TRN than in the PMv. The timing of activity modulations of visual- and movement-related neurons was similar in the TRNrd and PMv neurons. These results indicated that TRN neurons whose activity is modulated in parallel with PMv neurons, send spatially non-selective, inhibitory signals to the thalamic relay nuclei while receiving visual signals and preparing for and executing movements.

\section{The TRN sector projecting disynaptically to the PMv}

Using the trans-synaptic transport of rabies virus, we identified a sector of the TRN that projected to the PMv in a disynaptic manner. The rabies virus injected into the PMv is considered to be transferred retrogradely to the TRN, primarily via neurons in the thalamic relay nuclei, such as the $\mathrm{X}$ and VLo, which send direct projections

TABLE 5. Lead time, timing of activity peak, and peak firing rates of spatially selective movement-related neurons in the TRNrd and PMv

\begin{tabular}{|c|c|c|c|c|c|c|}
\hline \multirow[b]{2}{*}{ Target type } & \multicolumn{3}{|l|}{ Movement onset } & \multicolumn{3}{|l|}{ Touch } \\
\hline & Lead time (ms) & $\begin{array}{l}\text { Timing of } \\
\text { activity } \\
\text { peak (ms) }\end{array}$ & $\begin{array}{l}\text { Peak firing } \\
\text { rate } \\
\text { (spikes/s) }\end{array}$ & Lead time (ms) & $\begin{array}{l}\text { Timing of } \\
\text { activity } \\
\text { peak }(\mathrm{ms})\end{array}$ & $\begin{array}{l}\text { Peak firing } \\
\text { rate (spikes/s) }\end{array}$ \\
\hline \multicolumn{7}{|l|}{ TRN } \\
\hline Preferred target & $-119(-289 \pm 80)$ & 227 & 76.4 & $-444(-424 \pm 17)$ & -86 & 75.3 \\
\hline Non-preferred target & $-133(-858 \pm 55)$ & -59 & 63.7 & $-490(-425 \pm 23)$ & -350 & 62.8 \\
\hline \multicolumn{7}{|l|}{$\mathrm{PMv}$} \\
\hline Preferred target & $-218(-291 \pm 36)$ & 118 & 30.1 & $-455(-313 \pm 16)$ & -85 & 32.1 \\
\hline Non-preferred target & $-232(-354 \pm 40)$ & 140 & 21.7 & $-499(-393 \pm 18)$ & -103 & 21.4 \\
\hline
\end{tabular}

Numbers indicate the timings of population activity (ms) and the peak firing rate (spikes/s). Numbers in parentheses indicate means \pm standard error of means (ms) of single neurons. 
to the PMv (Fig. 2C and D) (Rouiller et al., 1999). Neurons in these nuclei receive major inputs from the cerebellum (to the $\mathrm{X}$ ) and basal ganglia (to the VLo) (Schell \& Strick, 1984; Sakai et al., 1996). Altogether, the present results suggest that the TRN is involved in modulating the information flow from the cerebellum and basal ganglia to the cerebral cortex via thalamus (Fig. 7). The neurons labeled in the TRN were found rostrocaudally, in the rostral to middle part, and dorsoventrally, in the dorsal to middle part of the TRN. The TRN sector (the TRNrd) defined in this study corresponds largely to the region that receives inputs from the cortical motor areas (Chow, 1952; Schell \& Strick, 1984; Rouiller et al., 1998). Additionally, the TRNrd seems to overlap with the region that receives inputs from the prefrontal cortex and amygdala (Zikopoulos \& Barbas, 2006, 2012). This suggests that the TRNrd receives not only motor signals from cortical motor areas, such as the PMv, but also cognitive/emotional signals from the prefrontal cortex (Boussaoud \& Wise, 1993) and the amygdala (Nishijo et al., 1988; Adolphs et al., 1994).

Although we identified the TRN sector (TRNrd) that sends disynaptic output to the PMv and characterized neurons in the TRNrd and PMv, we did not identify the direct synaptic connectivity between the PMv and the TRNrd. Thus, we only compared the

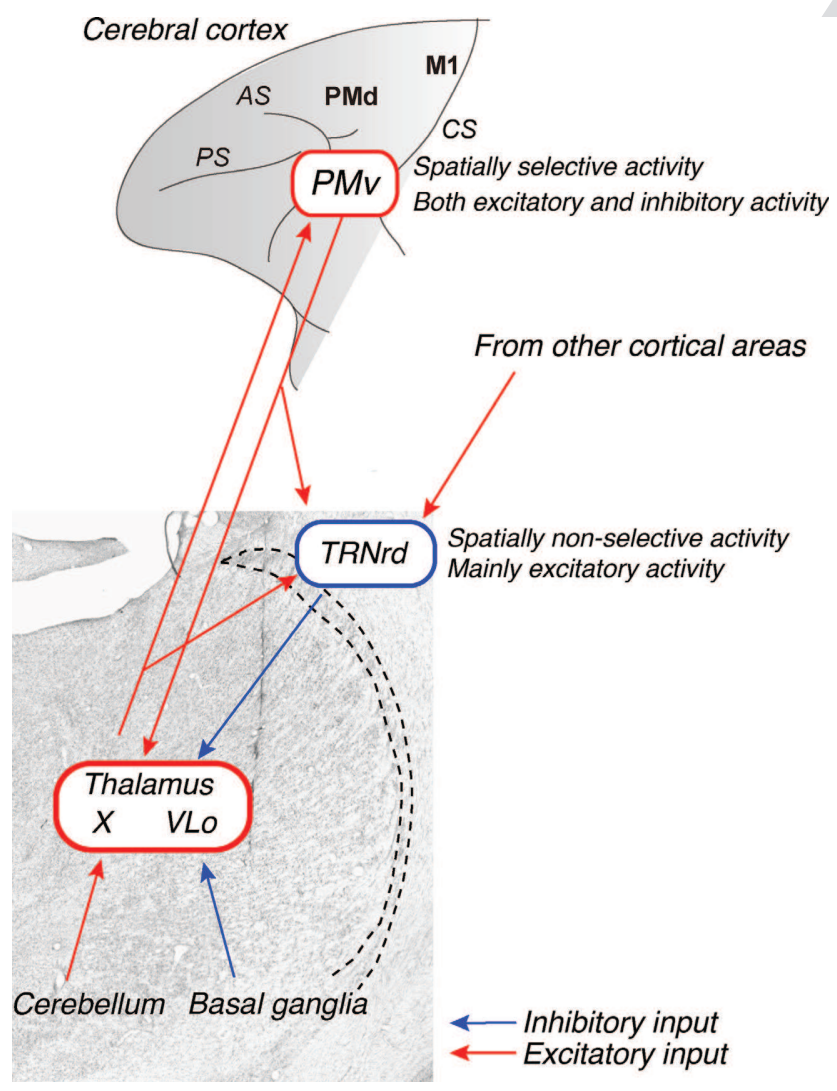

FIG. 7. Summary diagram showing the cortico-thalamic circuits around the TRN and subcortical structures. The PMv projects to the motor thalamic nuclei (X and VLo) and TRN, while the motor thalamic nuclei projects to the PMv and TRN. The TRN in turn sends inhibitory outputs to the motor thalamic nuclei. As the motor thalamic nuclei receives excitatory inputs from the cerebellum and inhibitory inputs from the basal ganglia, the TRN is considered to modulate the information flow from the cerebellum and the basal ganglia to the cerebral cortex. In this study, we analyzed activity of PMv and TRN neurons and found that the TRN and PMv are involved in the processing of visuomotor signals in an area-specific manner. Blue and red arrows indicate inhibitory and excitatory signals respectively. functional roles of the PMv vs. TRNrd neurons at an areal level. To get a deeper insight into the functional interactions between them, it is necessary to employ alternative approaches. In this context, Halassa et al. (2014) employed an optogenetic approach to identify TRN neurons receiving inputs from other thalamic nuclei. By recording neuronal activity from the TRN and other thalamic nuclei, they have shown that their interactions dynamically change according to the sleep-arousal states and the TRN neuron types, that is, sensory-projecting TRN neurons and limbic-projecting TRN neurons. Wimmer et al. (2015) also applied an optogentic approach to identify inputs to TRN and thalamic neurons from the prefrontal cortex and also to intercept inputs to TRN neurons from the prefrontal cortex and the visual cortex. They have demonstrated that TRN neurons receiving inputs from the prefrontal cortex dynamically modulate the activity depending on whether the animals attend to the visual or auditory stimuli. This reveals that the prefrontal cortex exerts top-down control on biased attention via the TRN. In addition, in the future it would be of great interest to identify inputs to single TRNrd neurons from multiple cortical areas (i.e. convergent inputs) by electrical or optical stimulation since Zikopoulos \& Barbas (2006) have reported that a single TRN sector receives converging inputs from diverse cortical areas.

\section{Visual-related neurons in TRNrd are characterized by spatially non-selective activity and activity increase}

We found that $47 \%$ of task-related neurons in the motor TRNrd showed visual-related activity. The fraction of spatially non-selective visual-related neurons was greater in the TRNrd $(81 \%)$ than in the PMv $(46 \%)$. In these non-selective neurons, the fraction of neurons with activity increase was greater in the TRNrd $(91 \%)$ than in the PMv (73\%). Moreover, the timing of activity increases in the TRNrd and PMv were fast and similar ( $100 \mathrm{~ms}$ after target onset). These results showed that the visual-related TRNrd neurons sent inhibitory signals to neurons in the thalamic relay nuclei each time motor targets appeared.

In terms of sensory responses, Shosaku (1985) reported that the somatosensory sector of the rat TRN displayed less directional selectivity in vibrissa-responding neurons and that the receptive field was larger in the TRN than in the somatosensory thalamic nuclei (Pinault, 2004). This less-precise representation of sensory signals is consistent with our findings. In the visual sector of the monkey TRN, McAlonan et al. (2008) showed that the activity of TRN neurons decreased when the monkeys attended to a visual stimulus presented in the receptive field and ignored another visual stimulus presented elsewhere (in visual modality attention), while the activity of neurons in the lateral geniculate nucleus (LGN) increased. These findings indicate that the visual sector of the TRN is involved in the attentional gating of visual signals (Crick, 1984). In cross-modal attention involving visual and auditory signals, neurons in the visual TRN showed enhanced activity when monkeys attended to visual signals rather than to auditory signals (McAlonan et al., 2006). Furthermore, functional deficits in TRN neurons induced by manipulating gene expression in the TRN resulted in abnormalities in attention (Ahrens et al., 2015). These findings indicate that the TRN is involved in the attentional processing of sensory signals, by increasing and decreasing inhibitory output signals to the thalamic relay nuclei. Together with the results of the prior studies, the results of this study showing that visual-related TRNrd neurons send spatially non-selective, inhibitory signals to the thalamic relay nuclei suggest that these signals may play an important role as an attentional gateway by conveying stronger representations and eliminating weaker ones. 


\section{Y. Saga et al.}

\section{Set- and movement-related neurons in TRNrd tend to show spatially non-selective activity and activity increase}

TRNrd neurons showed set- and movement-related activity, as described previously in cortical motor areas, including the PMv, and the motor thalamus (Tanji \& Kurata, 1982; Weinrich \& Wise, 1982; Godschalk et al., 1985; Kurata \& Wise, 1988; Alexander \& Crutcher, 1990a,b; Boussaoud \& Wise, 1993; di Pellegrino \& Wise, 1993; Crammond \& Kalaska, 1994; Shen \& Alexander, 1997; Cisek et al., 2003; Hoshi et al., 2005; Hoshi \& Tanji, 2006). Previous studies have stressed that neurons in the cortical motor areas and the motor thalamus encode spatial or directional aspects of movement, which is considered important for selecting and specifying actions (Jones, 1975; Weinrich \& Wise, 1982; Wise, 1985; Crutcher \& Alexander, 1990; Kalaska \& Crammond, 1992; Boussaoud \& Wise, 1993; Kurata, 1993, 2005; Johnson et al., 1996; Kalaska et al., 1997; Shen \& Alexander, 1997; Crammond \& Kalaska, 2000; Wyder et al., 2003; Cisek \& Kalaska, 2005; Nakayama et al., 2008; Kunimatsu \& Tanaka, 2010). In the present study, we found that the fraction of spatially non-selective, set-related neurons was greater in the TRNrd (93\% of set-related neurons) than in the PMv (60\%). Of these, $84 \%$ of TRNrd neurons showed an activity increase, whereas a smaller number of PMv neurons (59\%) did so. In the movementrelated neurons, the fraction of spatially non-selective neurons in the TRNrd (69\%) did not differ from that in the PMv (59\%). However, among them, the fraction of neurons with activity increase was greater in the TRNrd (84\%) than in the PMv (59\%), as with the visual-related and set-related neurons. These results indicate that along with the visual-related neurons, the set-related and movementrelated neurons in the TRNrd send spatially non-selective, inhibitory signals to the thalamic relay nuclei. These signals may be useful in sculpting set-related activity, eliminating unchosen motor signals, setting a threshold for set-related activity to gain effect, and suppressing a movement until a timely initiation.

\section{A subset of movement-related neurons in TRNrd show spatially selective activity increase}

In contrast with the visual- and set-related neurons, the fraction of spatially selective movement-related neurons in the TRNrd (31\%) did not differ from that in the PMv (41\%), and the timing of activity modulation was similar. In cats, movement-related activity of neurons in the motor sector of the TRN has been shown during locomotion (Marlinski et al., 2012; Marlinski \& Beloozerova, 2014). The TRN activity was modulated depending on the required locomotion type (i.e. simple vs. ladder locomotion). Thus, such movement-related activity is considered to actively encode the direction of a reaching movement and the type of locomotion. Our results suggest that the TRNrd may play an important role in specifying the movement direction or spatial information when monkeys make reaching movements.

\section{Theoretical considerations}

The TRN receives inputs from the thalamic nuclei and neurons in layers VI and V of the cortical areas (Guillery \& Harting, 2003; Zikopoulos \& Barbas, 2006, 2012; Sherman \& Guillery, 2011). Thus, the TRN receives feedback modulation from layer VI neurons and a feedforward driver from layer $\mathrm{V}$ neurons (Zikopoulos \& Barbas, 2006). We found that the timing of visual- and movementrelated neurons was similar in the TRNrd and PMv. This may suggest that the TRNrd receives feedforward driver inputs concerning visual and motor responses from cortical areas. Of the movement-related activity, $31 \%$ showed direction selectivity. Such a directional movement signal may also be carried by the feedforward driver signal from the cortical motor areas, including the PMv. The feedback modulation inputs may also contribute to generating spatially non-selective activity or form the background or baseline activity of TRN neurons. It would be of interest to determine the functional roles played by these two types of cortical input. With respect to the interaction between the PMv and the TRNrd, there are two additional possibilities. One possibility is that non-spatially selective PMv neurons preferentially project to the TRN, and the other is that PMv neurons with distinct spatial positions send converging inputs to single TRN neurons. These different mechanisms may underlie the non-spatially selective activity of TRNrd neurons.

Another important anatomical issue concerns local circuits. It has been proposed that TRN neurons have intrinsic connections with other TRN neurons, which may constitute a neural basis of lateral inhibition within the nucleus (Cox et al., 1996). We found that high-baseline discharges were suppressed at several time points, such as after visual cue onset, during preparation for movement, and during execution of movement. These findings suggest that the activity decrease, as well as any activity increase, may contribute to information processing by the TRN; furthermore, local circuits within the TRN, as well as interneurons in the thalamic relay nuclei, may also be involved in these processes. In relation to the lateral inhibition within the TRN, a recent study in a rodent has shown that the TRN intrinsic connection is functional only during a short period after birth (Hou et al., 2016). This finding is in line with a prior report showing that the lateral inhibition between TRN neurons is not so substantial (Pinault et al., 1997). This important issue should further be investigated in the TRN of nonhuman primate. Moreover, an intrinsic connection has been shown to mediate the interaction between first- and higher order thalamic relay nuclei (Pinault, 2004; Lam \& Sherman, 2007) and between nuclei representing different sensory modalities (Crabtree et al., 1998; Crabtree, 1999; Crabtree \& Isaac, 2002). Thus, it is possible that the TRNrd mediates the interaction between sensory and motor signals. In fact, we found that single TRNrd neurons exhibited visual- and movement-related activity. Another possibility is that the TRNrd mediates the interaction between the primary motor cortex and higher order motor areas such as the PMv. Multiple cortical motor areas are known to project to the rostro-dorsal sector of the TRN, corresponding to the TRNrd examined in the present study (Rouiller et al., 1998). Furthermore, inputs from the prefrontal cortex and inferotemporal cortex converge in the TRN (Zikopoulos \& Barbas, 2006). These findings indicate that the TRN may contribute to the integration of multiple systems, such as the PMv and the primary motor cortex, by collecting inputs from multiple cortical areas and by specific interaction mechanisms formed with thalamic relay nuclei.

TRN neurons use GABA as their neurotransmitter, indicating that they exert a suppressive action on the thalamic relay neurons. Previous studies showed that modulating neuronal activity of the TRN by microinjection of GABA-related substances resulted in changes in the size of the receptive fields of neurons in the thalamic relay nuclei (Sillito \& Kemp, 1983; Hicks et al., 1986; Lee et al., 1994a, b; Cotillon-Williams et al., 2008). In a similar manner, the TRN may be involved in sharpening the direction selectivity of set- and movement-related activity in neurons in the motor thalamic relay nuclei. One possibility is that the spatially non-selective activity functions as a filter and sets a threshold for passing stronger activity and for blocking weaker activity. Another possibility is that general suppression enhances the contrast of direction selectivity. Further, Pinault \& Deschenes (1998) showed anatomical evidence that TRN and thalamic neurons form open loop connections in the rat 
thalamus. Thus, a lateral inhibition mechanism may play an important role in the information processing in the thalamic relay nuclei.

A further possible function of the TRN concerns efference copies from cortical areas. Sherman \& Guillery (2011) showed that the TRN was involved in blocking efference copy signals from the cerebral cortex that were irrelevant for computations in the thalamic relay nuclei. The similar timing of activity modulation between the TRNrd and the PMv indicate that TRNrd can inhibit signals from the PMv at the level of the thalamic relay nuclei. Only the part of the motor-related activity issued by the PMv neurons that is needed in the thalamic relay nuclei may be transferred to them, while other signals may be suppressed.

In this study, we identified several features of TRN function. Further work based on these theoretical perspectives would enhance our understanding of the role(s) of the TRN in information processing through thalamo-cortical networks that are essential for almost all brain functions beyond sensory and motor aspects (Jones, 2007).

\section{Conflict of interest}

None of the authors has any competing financial interests.

\section{Acknowledgements}

The authors thank T. Ogata and T. Kuroda for technical assistance. This work was supported by AMED-CREST, AMED (to E. H. and M. T.), Japan Society for the Promotion of Science (JSPS) Postdoctoral Fellow for Research Abroad (to Y.S.), and Global COE Program of Tamagawa University (to Y.S., Y.N., T.Y., M.H., and E.H.).

\section{Abbreviations}

ICMS, intracortical microstimulation; LGN, lateral geniculate nucleus; M1, primary motor cortex; MD, mediodorsal nucleus; MT, movement time; PMd, dorsal premotor cortex; PMv, ventral premotor cortex; RF, receptive field; $\mathrm{RT}$, reaction time; TRN, thalamic reticular nucleus; VA, ventroanterior nucleus; VG task, visually guided reaching task; VLc, caudal division of ventrolateral nucleus; VLo, oral division of ventrolateral nucleus.

\section{Author contributions}

Y.S., Y.N., K.I., T.Y., M.H., L.T., M.T., and E.H. designed research; Y.S., Y.N., K.I., and T.Y., performed research; Y.S., and Y.N., analyzed data; Y.S., and E.H. wrote the paper.

\section{References}

Adolphs, R., Tranel, D., Damasio, H. \& Damasio, A. (1994) Impaired recognition of emotion in facial expressions following bilateral damage to the human amygdala. Nature, 372, 669-672.

Ahrens, S., Jaramillo, S., Yu, K., Ghosh, S., Hwang, G.R., Paik, R., Lai, C., He, M. et al. (2015) ErbB4 regulation of a thalamic reticular nucleus circuit for sensory selection. Nat. Neurosci., 18, 104-111.

Alexander, G.E. \& Crutcher, M.D. (1990a) Neural representations of the target (goal) of visually guided arm movements in three motor areas of the monkey. J. Neurophysiol., 64, 164-178.

Alexander, G.E. \& Crutcher, M.D. (1990b) Preparation for movement: neural representations of intended direction in three motor areas of the monkey. J. Neurophysiol., 64, 133-150.

Asanuma, C. (1994) GABAergic and pallidal terminals in the thalamic reticular nucleus of squirrel monkeys. Exp. Brain Res., 101, 439-451.

Boussaoud, D. \& Wise, S.P. (1993) Primate frontal cortex: effects of stimulus and movement. Exp. Brain Res., 95, 28-40.

Carman, J.B., Cowan, W.M. \& Powell, T.P. (1964) Cortical connexions of the thalamic reticular nucleus. J. Anat., 98, 587-598.

Chow, K.L. (1952) Regional degeneration of the thalamic reticular nucleus following cortical ablations in monkeys. J. Comp. Neurol., 97, 37-59.
Cicirata, F., Angaut, P., Serapide, M.F. \& Panto, M.R. (1990) Functional organization of the direct and indirect projection via the reticularis thalami nuclear complex from the motor cortex to the thalamic nucleus ventralis lateralis. Exp. Brain Res., 79, 325-337.

Cisek, P. \& Kalaska, J.F. (2005) Neural correlates of reaching decisions in dorsal premotor cortex: specification of multiple direction choices and final selection of action. Neuron, 45, 801-814.

Cisek, P., Crammond, D.J. \& Kalaska, J.F. (2003) Neural activity in primary motor and dorsal premotor cortex in reaching tasks with the contralateral versus ipsilateral arm. J. Neurophysiol., 89, 922-942.

Cotillon-Williams, N., Huetz, C., Hennevin, E. \& Edeline, J.M. (2008) Tonotopic control of auditory thalamus frequency tuning by reticular thalamic neurons. J. Neurophysiol., 99, 1137-1151.

Cox, C.L., Huguenard, J.R. \& Prince, D.A. (1996) Heterogeneous axonal arborizations of rat thalamic reticular neurons in the ventrobasal nucleus. J. Comp. Neurol., 366, 416-430.

Crabtree, J.W. (1999) Intrathalamic sensory connections mediated by the thalamic reticular nucleus. Cell. Mol. Life Sci., 56, 683-700.

Crabtree, J.W. \& Isaac, J.T. (2002) New intrathalamic pathways allowing modality-related and cross-modality switching in the dorsal thalamus. $J$. Neurosci., 22, 8754-8761.

Crabtree, J.W., Collingridge, G.L. \& Isaac, J.T. (1998) A new intrathalamic pathway linking modality-related nuclei in the dorsal thalamus. Nat. Neurosci., 1, 389-394.

Crammond, D.J. \& Kalaska, J.F. (1994) Modulation of preparatory neuronal activity in dorsal premotor cortex due to stimulus-response compatibility. J. Neurophysiol., 71, 1281-1284.

Crammond, D.J. \& Kalaska, J.F. (2000) Prior information in motor and premotor cortex: activity during the delay period and effect on pre-movement activity. J. Neurophysiol., 84, 986-1005.

Crick, F. (1984) Function of the thalamic reticular complex: the searchlight hypothesis. Proc. Natl Acad. Sci. USA, 81, 4586-4590.

Crutcher, M.D. \& Alexander, G.E. (1990) Movement-related neuronal activity selectively coding either direction or muscle pattern in three motor areas of the monkey. J. Neurophysiol., 64, 151-163.

Gandia, J.A., De Las Heras, S., Garcia, M. \& Gimenez-Amaya, J.M. (1993) Afferent projections to the reticular thalamic nucleus from the globus pallidus and the substantia nigra in the rat. Brain Res. Bull., 32, 351-358.

Godschalk, M., Lemon, R.N., Kuypers, H.G. \& van der Steen, J. (1985) The involvement of monkey premotor cortex neurones in preparation of visually cued arm movements. Behav. Brain Res., 18, 143-157.

Gonzalo-Ruiz, A. \& Lieberman, A.R. (1995a) GABAergic projections from the thalamic reticular nucleus to the anteroventral and anterodorsal thalamic nuclei of the rat. J. Chem. Neuroanat., 9, 165-174.

Gonzalo-Ruiz, A. \& Lieberman, A.R. (1995b) Topographic organization of projections from the thalamic reticular nucleus to the anterior thalamic nuclei in the rat. Brain Res. Bull., 37, 17-35.

Guillery, R.W. \& Harting, J.K. (2003) Structure and connections of the thalamic reticular nucleus: Advancing views over half a century. J. Comp. Neurol., 463, 360-371.

Guillery, R.W. \& Sherman, S.M. (2002) Thalamic relay functions and their role in corticocortical communication: generalizations from the visual system. Neuron, 33, 163-175.

Halassa, M.M., Chen, Z., Wimmer, R.D., Brunetti, P.M., Zhao, S., Zikopoulos, B., Wang, F., Brown, E.N. et al. (2014) State-dependent architecture of thalamic reticular subnetworks. Cell, 158, 808-821.

Hazrati, L.N. \& Parent, A. (1991) Projection from the external pallidum to the reticular thalamic nucleus in the squirrel monkey. Brain Res., 550, $142-146$.

Hicks, T.P., Metherate, R., Landry, P. \& Dykes, R.W. (1986) Bicucullineinduced alterations of response properties in functionally identified ventroposterior thalamic neurones. Exp. Brain Res., 63, 248-264.

Hoshi, E. \& Tanji, J. (2006) Differential involvement of neurons in the dorsal and ventral premotor cortex during processing of visual signals for action planning. J. Neurophysiol., 95, 3596-3616.

Hoshi, E., Sawamura, H. \& Tanji, J. (2005) Neurons in the rostral cingulate motor area monitor multiple phases of visuomotor behavior with modest parametric selectivity. J. Neurophysiol., 94, 640-656.

Hou, G., Smith, A.G. \& Zhang, Z.W. (2016) Lack of intrinsic GABAergic connections in the thalamic reticular nucleus of the mouse. J. Neurosci., 36, 7246-7252.

Houser, C.R., Vaughn, J.E., Barber, R.P. \& Roberts, E. (1980) GABA neurons are the major cell type of the nucleus reticularis thalami. Brain Res., 200, 341-354. 


\section{Y. Saga et al.}

Ishida, H., Inoue, K., Takada, M. \& Hoshi, E. (2016) Origins of multisynaptic projections from the basal ganglia to the forelimb region of the ventral premotor cortex in macaque monkeys. Eur. J. Neurosci., 43, 258-269.

Johnson, P.B., Ferraina, S., Bianchi, L. \& Caminiti, R. (1996) Cortical networks for visual reaching: physiological and anatomical organization of frontal and parietal lobe arm regions. Cereb. Cortex, 6, 102-119.

Jones, E.G. (1975) Some aspects of the organization of the thalamic reticular complex. J. Comp. Neurol., 162, 285-308.

Jones, E.G. (2007) The Thalamus. 2nd Edn, Cambridge University Press, Cambridge, UK.

Kalaska, J.F. \& Crammond, D.J. (1992) Cerebral cortical mechanisms of reaching movements. Science, 255, 1517-1523.

Kalaska, J.F., Scott, S.H., Cisek, P. \& Sergio, L.E. (1997) Cortical control of reaching movements. Curr. Opin. Neurobiol., 7, 849-859.

Kelly, R.M. \& Strick, P.L. (2000) Rabies as a transneuronal tracer of circuits in the central nervous system. J. Neurosci. Methods, 103, 63-71.

Kunimatsu, J. \& Tanaka, M. (2010) Roles of the primate motor thalamus in the generation of antisaccades. J. Neurosci., 30, 5108-5117.

Kurata, K. (1993) Premotor cortex of monkeys: set- and movement-related activity reflecting amplitude and direction of wrist movements. J. Neurophysiol., 69, 187-200.

Kurata, K. (2005) Activity properties and location of neurons in the motor thalamus that project to the cortical motor areas in monkeys. J. Neurophysiol., 94, 550-566.

Kurata, K. \& Hoffman, D.S. (1994) Differential effects of muscimol microinjection into dorsal and ventral aspects of the premotor cortex of monkeys. J. Neurophysiol., 71, 1151-1164.

Kurata, K. \& Hoshi, E. (2002) Movement-related neuronal activity reflecting the transformation of coordinates in the ventral premotor cortex of monkeys. J. Neurophysiol., 88, 3118-3132.

Kurata, K. \& Wise, S.P. (1988) Premotor cortex of rhesus monkeys: setrelated activity during two conditional motor tasks. Exp. Brain Res., 69, 327-343.

Lam, Y.W. \& Sherman, S.M. (2007) Different topography of the reticulothalmic inputs to first- and higher-order somatosensory thalamic relays revealed using photostimulation. J. Neurophysiol., 98, 2903-2909.

Lee, S.M., Friedberg, M.H. \& Ebner, F.F. (1994a) The role of GABAmediated inhibition in the rat ventral posterior medial thalamus. I. Assessment of receptive field changes following thalamic reticular nucleus lesions. J. Neurophysiol., 71, 1702-1715.

Lee, S.M., Friedberg, M.H. \& Ebner, F.F. (1994b) The role of GABAmediated inhibition in the rat ventral posterior medial thalamus. II. Differential effects of GABAA and GABAB receptor antagonists on responses of VPM neurons. J. Neurophysiol., 71, 1716-1726.

Marlinski, V. \& Beloozerova, I.N. (2014) Burst firing of neurons in the thalamic reticular nucleus during locomotion. J. Neurophysiol., 112, 181-192.

Marlinski, V., Sirota, M.G. \& Beloozerova, I.N. (2012) Differential gating of thalamocortical signals by reticular nucleus of thalamus during locomotion. J. Neurosci., 32, 15823-15836.

Matelli, M., Luppino, G. \& Rizzolatti, G. (1985) Patterns of cytochrome oxidase activity in the frontal agranular cortex of the macaque monkey. Behav. Brain Res., 18, 125-136.

McAlonan, K., Cavanaugh, J. \& Wurtz, R.H. (2006) Attentional modulation of thalamic reticular neurons. J. Neurosci., 26, 4444-4450.

McAlonan, K., Cavanaugh, J. \& Wurtz, R.H. (2008) Guarding the gateway to cortex with attention in visual thalamus. Nature, 456, 391-394.

McCormick, D.A. (1992) Neurotransmitter actions in the thalamus and cerebral cortex and their role in neuromodulation of thalamocortical activity. Prog. Neurobiol., 39, 337-388.

Morrison, J.H. \& Foote, S.L. (1986) Noradrenergic and serotoninergic innervation of cortical, thalamic, and tectal visual structures in Old and New World monkeys. J. Comp. Neurol., 243, 117-138.

Nakayama, Y., Yamagata, T., Tanji, J. \& Hoshi, E. (2008) Transformation of a virtual action plan into a motor plan in the premotor cortex. J. Neurosci., 28, 10287-10297.

Nakayama, Y., Yamagata, T. \& Hoshi, E. (2016) Rostrocaudal functional gradient among the pre-dorsal premotor cortex, dorsal premotor cortex, and primary motor cortex in goal-directed motor behavior. Eur. J. Neurosci., 43, 1569-1589.

Nishijo, H., Ono, T. \& Nishino, H. (1988) Single neuron responses in amygdala of alert monkey during complex sensory stimulation with affective significance. J. Neurosci., 8, 3570-3583.

Nomoto, K., Schultz, W., Watanabe, T. \& Sakagami, M. (2010) Temporally extended dopamine responses to perceptually demanding reward-predictive stimuli. J. Neurosci., 30, 10692-10702.
Pare, D., Hazrati, L.N., Parent, A. \& Steriade, M. (1990) Substantia nigra pars reticulata projects to the reticular thalamic nucleus of the cat: a morphological and electrophysiological study. Brain Res., 535, 139-146.

di Pellegrino, G. \& Wise, S.P. (1993) Visuospatial versus visuomotor activity in the premotor and prefrontal cortex of a primate. J. Neurosci., 13, 1227-1243.

Pinault, D. (2004) The thalamic reticular nucleus: structure, function and concept. Brain Res. Rev., 46, 1-31.

Pinault, D. \& Deschenes, M. (1998) Projection and innervation patterns of individual thalamic reticular axons in the thalamus of the adult rat: a three-dimensional, graphic, and morphometric analysis. J. Comp. Neurol., 391, 180-203.

Pinault, D., Smith, Y. \& Deschenes, M. (1997) Dendrodendritic and axoaxonic synapses in the thalamic reticular nucleus of the adult rat. J. Neurosci., 17, 3215-3233.

Pollin, B. \& Rokyta, R. (1982) Somatotopic organization of nucleus reticularis thalami in chronic awake cats and monkeys. Brain Res., 250, 211-221.

Rouiller, E.M., Tanne, J., Moret, V., Kermadi, I., Boussaoud, D. \& Welker, E. (1998) Dual morphology and topography of the corticothalamic terminals originating from the primary, supplementary motor, and dorsal premotor cortical areas in macaque monkeys. J. Comp. Neurol., 396, 169-185.

Rouiller, E.M., Tanne, J., Moret, V. \& Boussaoud, D. (1999) Origin of thalamic inputs to the primary, premotor, and supplementary motor cortical areas and to area 46 in macaque monkeys: a multiple retrograde tracing study. J. Comp. Neurol., 409, 131-152.

Saga, Y., Hirata, Y., Takahara, D., Inoue, K., Miyachi, S., Nambu, A., Tanji, J., Takada, M. et al. (2011) Origins of multisynaptic projections from the basal ganglia to rostrocaudally distinct sectors of the dorsal premotor area in macaques. Eur. J. Neurosci., 33, 285-297.

Saga, Y., Hashimoto, M., Tremblay, L., Tanji, J. \& Hoshi, E. (2013) Representation of spatial- and object-specific behavioral goals in the dorsal globus pallidus of monkeys during reaching movement. J. Neurosci., 33, 16360-16371.

Sakai, S.T., Inase, M. \& Tanji, J. (1996) Comparison of cerebellothalamic and pallidothalamic projections in the monkey (Macaca fuscata): a double anterograde labeling study. J. Comp. Neurol., 368, 215-228.

Schell, G.R. \& Strick, P.L. (1984) The origin of thalamic inputs to the arcuate premotor and supplementary motor areas. J. Neurosci., 4, 539-560.

Shen, L. \& Alexander, G.E. (1997) Neural correlates of a spatial sensory-to-motor transformation in primary motor cortex. J. Neurophysiol., 77, 1171-1194.

Sherman, S.M. \& Guillery, R.W. (2011) Distinct functions for direct and transthalamic corticocortical connections. J. Neurophysiol., 106, 1068-1077.

Sillito, A.M. \& Kemp, J.A. (1983) The influence of GABAergic inhibitory processes on the receptive field structure of $\mathrm{X}$ and $\mathrm{Y}$ cells in cat dorsal lateral geniculate nucleus (dLGN). Brain Res., 277, 63-77.

Steriade, M. (2005) Sleep, epilepsy and thalamic reticular inhibitory neurons. Trends Neurosci., 28, 317-324.

Takahara, D., Inoue, K.I., Hirata, Y., Miyachi, S., Nambu, A., Takada, M. \& Hoshi, E. (2012) Multisynaptic projections from the ventrolateral prefrontal cortex to the dorsal premotor cortex in macaques - anatomical substrate for conditional visuomotor behavior. Eur. J. Neurosci., 36, 33653375 .

Tanji, J. \& Kurata, K. (1982) Comparison of movement-related activity in two cortical motor areas of primates. J. Neurophysiol., 48, 633-653.

Weinrich, M. \& Wise, S.P. (1982) The premotor cortex of the monkey. J. Neurosci., 2, 1329-1345.

Wimmer, R.D., Schmitt, L.I., Davidson, T.J., Nakajima, M., Deisseroth, K. \& Halassa, M.M. (2015) Thalamic control of sensory selection in divided attention. Nature, 526, 705-709.

Wise, S.P. (1985) The primate premotor cortex: past, present, and preparatory. Annu. Rev. Neurosci., 8, 1-19.

Wyder, M.T., Massoglia, D.P. \& Stanford, T.R. (2003) Quantitative assessment of the timing and tuning of visual-related, saccade-related, and delay period activity in primate central thalamus. J. Neurophysiol., 90, 20292052

Yamagata, T., Nakayama, Y., Tanji, J. \& Hoshi, E. (2009) Processing of visual signals for direct specification of motor targets and for conceptual representation of action targets in the dorsal and ventral premotor cortex. J. Neurophysiol., 102, 3280-3294.

Zikopoulos, B. \& Barbas, H. (2006) Prefrontal projections to the thalamic reticular nucleus form a unique circuit for attentional mechanisms. J. Neurosci., 26, 7348-7361.

Zikopoulos, B. \& Barbas, H. (2012) Pathways for emotions and attention converge on the thalamic reticular nucleus in primates. J. Neurosci., 32, $5338-5350$ 


\section{Graphical Abstract}

The contents of this page will be used as part of the graphical abstract of html only. It will not be published as part of main article.

\section{Visually guided task}
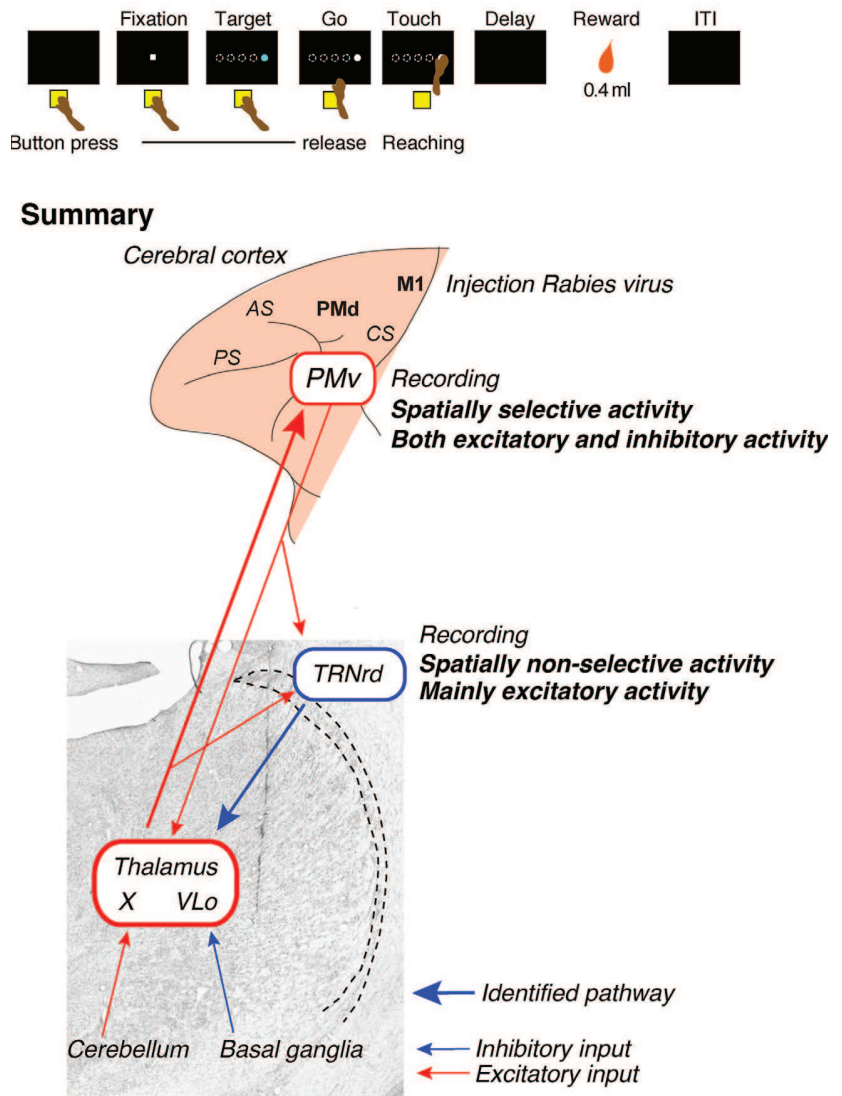

The rostro-dorsal sector of the thalamic reticular nucleus (TRNrd) projects via the motor thalamic nuclei, such as X and VLo, to the ventral premotor cortex (PMv). In monkeys performing a visually guided task, TRNrd and PMv neurons showed visual-, set-, and movement-related activity modulation. These results indicate that TRNrd modulates the information flow from the cerebellum and basal ganglia to the PMv via the motor thalamic nuclei for achieving visually guided reaching movements. 